The Fundamental Discussion between Newton, Maxwell, Bohr and Einstein

(version 4.0)

Wim Vegt

Department of Physics

Eindhoven University of Technology

5600-MB Eindhoven

The Netherlands.

E-mail:wimvegt@universitycollege.eu

\title{
Abstract
}

Isaac Newton, James Clerk Maxwell, Niels Bohr and Albert Einstein lived in fundamentally different time frames. Newton in the $16^{\text {th }}$ century, Maxwell in the $18^{\text {th }}$ century, Bohr in the $20^{\text {th }}$ century and Einstein was physically living in the $20^{\text {th }}$ century but he was his time far ahead and with his concept of a "curved space-time continuum" more connected to the $21^{\text {st }}$ century.

An interesting question would be: "Who would win the fundamental discussion about the interaction between "Gravity and Light" comparing the 4 fundamentally different time-frames? Newton, Maxwell, Bohr or Einstein? Newton with the fundamental " 3 rd law of equilibrium between the forces (force-densities)". Maxwell who had built the "Mathematical Foundation for Electrodynamics", Bohr (together with Heisenberg) who overruled Einstein during the $5^{\text {th }}$ Solvay Conference in 1927 with the fundamental concept of "Quantum Mechanical Probability" or Einstein (his time-frame far ahead) who postulated a "Curved SpaceTime Continuum" within a gravitational field.

It is still the question who was right? Newton, Maxwell, Bohr or Einstein? This article will discuss the interaction between "Gravity and Light" based on a deductive discussion based 
on the fundamental arguments and way of thinking within that corresponding time-frame.

Keywords: General Relativity; Quantum Physics; Dirac Equation; Gravitational-Electromagnetic Interaction; Black Holes; Gravitational-Electromagnetic Confinement; Electromagnetism; Quantum Optics 


\section{The Fundamental Discussion between Newton and Maxwell}

To find a new "Fundamental Electromagnetic Equation" to describe the Electromagnetic field (including Electromagnetic Interaction) in a different and more complete way than Maxwell did (Einstein called Maxwell the greatest physicist of his century), we have to go back to the time of Isaac Newton who published in 1687 in the "Philosophiae Naturalis Principia Mathematica" a Universal Fundamental Principle in Physics. "The Fundamental Principle of Harmony in the Whole Universe" based on his religious understanding of the "Creator of the Universe". Newton expressed this "Harmony" in the foundation of a "Universal Equilibrium" and he expressed the "Universal Equilibrium" in his third Law on which all Physics (till now) has been based.

\subsection{Newton's approach to Electrodynamics and} Electromagnetic Interaction based on Newtonian Physics.

Newton found the concept of "Universal Equilibrium" which he formulated in his famous third equation Action = Reaction. In nowadays math the concept of "Universal Equilibrium" has been formulated as:

$$
\sum_{i=0}^{i=n} \overline{\mathrm{F}_{\mathrm{i}}}=0
$$

Because the Inertia Force is a Reaction Force, the Inertia Force appears in the equation with a minus sign. 


$$
\sum_{i=0}^{i=n} \overline{\mathrm{F}_{\mathrm{i}}}-\mathrm{m} \overline{\mathrm{a}}=0
$$

Equation (2) is a general presentation of Newton's famous second law of motion. In a fundamental way, Newton's second law of motion describes the required electromagnetic equation for the GravitationalElectromagnetic Interaction in general terms, including Maxwell's theory of Electrodynamics published in 1865 in the article: "A Dynamic Theory of the Electromagnetic Field" and Einstein's theory of General Relativity published in 1911 the article: "On the Influence of Gravitation on the Propagation of Light".

Because Maxwell's 4 equations are not part of one whole uniform understanding of the universe like the fundamental equation of Newton's second law of motion represents, Maxwell's theory is missing the fundamental foundation.

Newton's second law of motion has been based on a profound understanding of the universe which is based on the fundamental principle of "Harmony and Equilibrium", expressed in equation (2).

To realize the new "Gravitational-Electromagnetic Equation", Newton's second law of motion will be the Universal Concept in Physics on which "Quantum Light Theory" will be built. The fundamental Electromagnetic force density equation has been based integral on Newton's second law of motion and has been divided into 5 separate terms (B-1 until B-5), each one describing a part of the electromagnetic and inertia force densities. 


$$
\sum_{i=0}^{i=5} \mathrm{~B}_{\mathrm{i}}=0
$$

The first term B-1 represents the inertia of the mass density of light (Electromagnetic Radiation). The terms B-2 and B-3 represent the electric force densities within the Electromagnetic Radiation (Beam of Light) and the terms B4 and B-5 represent the magnetic force densities within the Electromagnetic Radiation (Beam of Light).

Fundamental in the "Quantum Light Theory" is the outcome of (3) which always has to be zero according Newton's fundamental principle of "Universal Equilibrium".

To apply the concept of "Universal Equilibrium" within an electromagnetic field, the electric forces $F_{\text {Electric }}$, the magnetic forces $F_{\text {Magnetic }}$ and the inertia forces will be presented separately in equation (3):

$$
\sum_{i=0, j=0}^{i=n, j=m}\left(\overline{\mathrm{F}_{\text {Electric- }}}+\overline{\mathrm{F}_{\text {Magnetic-j }}}-\mathrm{m} \overline{\mathrm{a}}\right)=0
$$

\subsection{The Inertia of Light (Term B-1)}

Reducing Equation (2) to one single Force $\overline{\mathrm{F}}$, equation (2) will be written in the well-known presentation:

$$
\overline{\mathrm{F}}=\mathrm{m} \overline{\mathrm{a}}
$$

The right and the left term of Newton's law of motion in equation (5) has to be divided by the Volume "V" to find an 5 
equation for the force density $\bar{f}$ related to the mass density " $\rho$ ".

$$
\begin{aligned}
\overline{\mathrm{F}} & =\mathrm{m} \overline{\mathrm{a}} \\
\left(\frac{\overline{\mathrm{F}}}{\mathrm{V}}\right) & =\left(\frac{\mathrm{m}}{\mathrm{V}}\right) \overline{\mathrm{a}} \\
\overline{\mathrm{f}} & =\rho \overline{\mathrm{a}}
\end{aligned}
$$

The Inertia Force $\overline{\mathrm{F}_{\text {Inertia }}}$ for Electromagnetic Radiation will be derived from Newton's second law of motion, using the relationship between the momentum vector $\bar{p}$ for radiation expressed by the Poynting vector $\overline{\mathrm{S}}$ :

$$
\overline{\mathrm{F}_{\text {INERTIA }}}=-\mathrm{m} \overline{\mathrm{a}}=-\mathrm{m} \frac{\Delta \overline{\mathrm{v}}}{\Delta \mathrm{t}}=-\frac{\Delta(\mathrm{m} \overline{\mathrm{v}})}{\Delta \mathrm{t}}=-\frac{\Delta \overline{\mathrm{p}}}{\Delta \mathrm{t}}=-\left(\frac{V}{\mathrm{c}^{2}}\right) \frac{\Delta \overline{\mathrm{S}}}{\Delta \mathrm{t}}
$$

Dividing the right and the left term in equation (7) by the volume $\mathrm{V}$ results in the inertia force density $\overline{f_{\text {Inertia }}}$ :

$$
\begin{aligned}
& \overline{\mathrm{F}_{\text {INERTIA }}}=-\mathrm{m} \overline{\mathrm{a}}=-\mathrm{m} \frac{\Delta \overline{\mathrm{v}}}{\Delta \mathrm{t}}=-\frac{\Delta(\mathrm{m} \overline{\mathrm{v}})}{\Delta \mathrm{t}}=-\frac{\Delta \overline{\mathrm{p}}}{\Delta \mathrm{t}}=-\left(\frac{V}{\mathrm{c}^{2}}\right) \frac{\Delta \overline{\mathrm{S}}}{\Delta \mathrm{t}} \\
& \overline{\frac{\mathrm{F}_{\text {INERTIA }}}{\mathrm{V}}}=-\frac{\mathrm{m}}{V} \overline{\mathrm{a}}=-\frac{\mathrm{m}}{V} \frac{\Delta \overline{\mathrm{v}}}{\Delta \mathrm{t}}=-\frac{1}{V} \frac{\Delta \overline{\mathrm{p}}}{\Delta \mathrm{t}}=-\left(\frac{1}{\mathrm{c}^{2}}\right) \frac{\Delta \overline{\mathrm{S}}}{\Delta \mathrm{t}} \\
& \overline{\mathrm{f}_{\text {INERTIA }}}=-\rho \overline{\mathrm{a}}=-\left(\frac{1}{\mathrm{c}^{2}}\right) \frac{\Delta \overline{\mathrm{S}}}{\Delta \mathrm{t}}\left[\mathrm{N} / \mathrm{m}^{3}\right]
\end{aligned}
$$

The Poynting vector $\overline{\mathrm{S}}$ represents the total energy transport of the electromagnetic radiation per unit surface per unit time [ $\mathrm{J}$ $\left./ \mathrm{m}^{2} \mathrm{~s}\right]$. Which can be written as the cross product of the Electric Field intensity $\overline{\mathrm{E}}$ and the magnetic Field intensity $\overline{\mathrm{H}}$. 


$$
\begin{aligned}
& \overline{\mathrm{f}_{\text {INERTIA }}}=-\rho \mathrm{a}=-\left(\frac{1}{\mathrm{c}^{2}}\right) \frac{\Delta \mathrm{S}}{\Delta \mathrm{t}}=-\left(\frac{1}{\mathrm{c}^{2}}\right) \frac{\Delta(\overline{\mathrm{E}} \times \overline{\mathrm{H}})}{\Delta \mathrm{t}}\left[\mathrm{N} / \mathrm{m}^{3}\right] \\
& \overline{\mathrm{f}_{\text {INERTIA }}}=-\left(\frac{1}{\mathrm{c}^{2}}\right) \frac{\partial(\overline{\mathrm{E}} \times \overline{\mathrm{H}})}{\partial \mathrm{t}}\left[\mathrm{N} / \mathrm{m}^{3}\right]
\end{aligned}
$$

\subsection{Coulomb's Law (Colomb Force) for Electromagnetic GEONs (Term B-2 and B-4)}

An example of the Coulomb Force is the Electric Force $F_{\text {Coulomb }}$ acting on an electric charge $Q$ placed in an electric field $\mathrm{E}$. The equation for the Coulomb Force equals:

$$
\overline{\mathrm{F}_{\text {Coulomb }}}=\overline{\mathrm{E}} \mathrm{Q} \quad[\mathrm{N}]
$$

Dividing the right and the left term in equation (10) by the volume $\mathrm{V}$ results in the Electric force density $\overline{f_{\text {Coulomb }}}$ :

$$
\begin{aligned}
& \overline{\mathrm{F}}_{\text {COUlomb }}=\overline{\mathrm{E}} \mathrm{Q}[\mathrm{N}] \\
& \frac{\overline{\mathrm{F}}_{\text {COUlOmB }}}{\mathrm{V}}=\overline{\mathrm{E}} \frac{\mathrm{Q}}{\mathrm{V}}\left[\mathrm{N} / \mathrm{m}^{3}\right] \\
& \overline{\mathrm{f}}_{\text {COUlomb }}=\overline{\mathrm{E}} \rho_{\mathrm{E}}\left[\mathrm{N} / \mathrm{m}^{3}\right]
\end{aligned}
$$

Substituting Gauss's law in differential form in (11) results in Coulombs Law for Electromagnetic Radiation for the Electric force density $\overline{\mathrm{f}_{\text {Coulomb }} \text { : }}$ 


$$
\begin{aligned}
& \overline{\mathrm{f}}_{\text {солlomb }}=\overline{\mathrm{E}} \rho_{\mathrm{E}} \\
& \overline{\mathrm{f}}_{\text {Coulomb }}=\overline{\mathrm{E}} \rho_{\mathrm{E}}=\overline{\mathrm{E}}(\nabla \cdot \overline{\mathrm{D}}) \\
& \overline{\mathrm{f}}_{\text {Coulomb }}=\overline{\mathrm{E}}(\nabla \cdot \mathrm{D})=\varepsilon \overline{\mathrm{E}}(\nabla \cdot \overline{\mathrm{E}})\left[\mathrm{N} / \mathrm{m}^{3}\right]
\end{aligned}
$$

In Electromagnetic Field Configurations, there is in general no preference for the electric force densities or the magnetic force densities. In general the equations for the electric field densities are universally exchangeable with the magnetic field densities.

For the magnetic field densities, equation (12) can be written as:

$$
\begin{aligned}
& \overline{\mathrm{f}}_{\text {Coulomb - Electric }}=\overline{\mathrm{E}}(\nabla \cdot \overline{\mathrm{D}})=\varepsilon \overline{\mathrm{E}}(\nabla \cdot \overline{\mathrm{E}})\left[\mathrm{N} / \mathrm{m}^{3}\right](\text { Term B-2) } \\
& \overline{\mathrm{f}}_{\text {Coulomb - Magnetic }}=\overline{\mathrm{H}}(\nabla \cdot \overline{\mathrm{B}})=\mu \overline{\mathrm{H}}(\nabla \cdot \overline{\mathrm{H}})\left[\mathrm{N} / \mathrm{m}^{3}\right](\text { Term B-4) }
\end{aligned}
$$

\subsection{Lorentz's Law (Lorentz Force) for Electromagnetic GEONs (Term B-3 and B-5)}

An example of the Lorentz Force is the Magnetic Force $\mathrm{F}_{\text {Lorentz }}$ acting on an electric charge $\mathrm{Q}$ moving with a velocity $\mathrm{v}$ within a magnetic field with magnetic field intensity $\mathrm{B}$ (magnetic induction). 


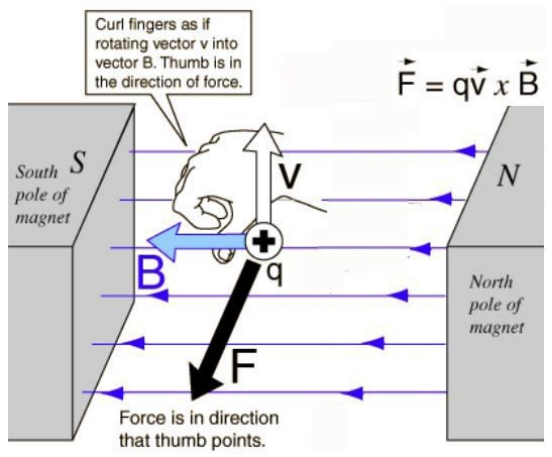

Fig. 1. The Lorentz Force equals the cross product of the Magnetic Induction $B$ and the velocity $v$ of the charge $q$ moving within the magnetic field times the value of the electric charge

The equation for the Lorentz Force equals:

$$
\overline{\mathrm{F}}_{\text {LORENTZ }}=\mathrm{Q} \overline{\mathrm{v}} \times \overline{\mathrm{B}}[\mathrm{N}]
$$

Dividing the right and the left term in equation (14) by the volume $V$ results in the Lorentz force density $\overline{f_{\text {Lorentz }}}$

$$
\begin{aligned}
& \overline{\mathrm{F}}_{\text {LORENTZ }}=\mathrm{Q} \overline{\mathrm{v}} \times \overline{\mathrm{B}}[\mathrm{N}] \\
& \frac{\overline{\mathrm{F}}_{\text {LORENTZ }}}{\mathrm{V}}=-\overline{\mathrm{B}} \times \frac{\mathrm{Q} \overline{\mathrm{v}}}{\mathrm{V}}\left[\mathrm{N} / \mathrm{m}^{3}\right] \\
& \overline{\mathrm{f}}_{\text {LORENTZ }}=-\overline{\mathrm{B}} \times \frac{\mathrm{Q} \overline{\mathrm{v}}}{\mathrm{V}}=-\overline{\mathrm{B}} \times \overline{\mathrm{j}}=-\mu \overline{\mathrm{H}} \times \overline{\mathrm{j}}\left[\mathrm{N} / \mathrm{m}^{3}\right]
\end{aligned}
$$

In which $q$ is the electric charge, $v$ the velocity of the electric charge, $B$ the magnetic induction and $j$ the electric current density. Substituting Ampère's law in differential form in 
(15) results in Lorentz's Law for Electromagnetic Radiation for the Electric force density $\overline{\mathrm{f}_{\text {Lorentz }}}$ :

$\overline{\mathrm{f}}_{\text {LORENTZ }}=-\mu \overline{\mathrm{H}} \times(\overline{\mathrm{j}})$

$\overline{\mathrm{f}}_{\text {LORENTZ }}=-\mu \overline{\mathrm{H}} \times(\overline{\mathrm{j}})=-\mu \overline{\mathrm{H}} \times(\nabla \times \overline{\mathrm{H}})\left[\mathrm{N} / \mathrm{m}^{3}\right]$

In Electromagnetic Field Configurations, there is in general no preference for the electric force densities or the magnetic force densities. In general the equations for the electric field densities are universally exchangeable with the magnetic field densities. For the electric field densities, equation (16) can be written as:

$$
\begin{aligned}
& \overline{\mathrm{f}}_{\text {Coulomb - Electric }}=-\varepsilon \bar{E} \times(\nabla \times \bar{E})\left[\mathrm{N} / \mathrm{m}^{3}\right](\text { Term B-3 }) \\
& \overline{\mathrm{f}}_{\text {Coulomb - Magnetic }}=-\mu \overline{\mathrm{H}} \times(\nabla \times \overline{\mathrm{H}})\left[\mathrm{N} / \mathrm{m}^{3}\right](\text { Term B-5 })
\end{aligned}
$$




\subsection{The Universal Equation for the Electromagnetic field (Term B-1 + Term B-2 + Term B-3 + Term B-4 + Term B-5)}

Newton's second law of motion applied within any arbitrary electromagnetic field configuration results in the fundamental equation (23) for any arbitrary electromagnetic field configuration (a beam of light):

$$
\begin{aligned}
& \text { NEWTON: } \mathrm{F}_{\text {TOTAAL }}=\text { ma represents: } \mathrm{f}_{\text {TOTAAL }}=\rho \mathrm{a} \\
& \begin{array}{llllll}
-\rho \mathrm{a} & + & \multicolumn{5}{c}{\mathrm{f}_{\text {TOTAAL }}} & =0 \\
-\rho \mathrm{a} & + & \mathrm{f}_{\text {ELEKTRISCH }} & + & \mathrm{f}_{\text {MAGNETISCH }} & =0 \\
-\rho \mathrm{a} & +\mathrm{F}_{\text {COULOMB }}+\mathrm{F}_{\text {LORENTZ }}+\mathrm{F}_{\text {COULOMB }}+\mathrm{F}_{\text {LORENTZ }} & =0
\end{array} \\
& -\frac{1}{c^{2}} \frac{\partial(\overline{\mathrm{E}} \times \overline{\mathrm{H}})}{\partial t}+\varepsilon_{0} \overline{\mathrm{E}}(\nabla \cdot \overline{\mathrm{E}})-\varepsilon_{0} \overline{\mathrm{E}} \times(\nabla \times \overline{\mathrm{E}})+\mu_{0} \overline{\mathrm{H}}(\nabla \cdot \overline{\mathrm{H}})-\mu_{0} \overline{\mathrm{H}} \times(\nabla \times \overline{\mathrm{H}})=0
\end{aligned}
$$

Term B-4 is the magnetic equivalent of the (electric) Coulomb's law B-2 and Term B-3 is the electric equivalent of the (magnetic) Lorentz's law B-5.

The universal equation for the electromagnetic field (free electromagnetic waves and confined electromagnetic fields) has been presented in (24) and expresses the perfect equilibrium between the inertia forces (B-1), the electric forces (B-2 and B-3) and the magnetic forces (B-4 and B-5) in any arbitrary electromagnetic field configuration.

$$
\begin{gathered}
-\frac{1}{c^{2}} \frac{\partial(\overline{\mathrm{E}} \times \overline{\mathrm{H}})}{\partial t}+\varepsilon_{0} \overline{\mathrm{E}}(\nabla . \overline{\mathrm{E}})-\varepsilon_{0} \overline{\mathrm{E}} \times(\nabla \times \overline{\mathrm{E}})+\mu_{0} \overline{\mathrm{H}}(\nabla \cdot \overline{\mathrm{H}})-\mu_{0} \overline{\mathrm{H}} \times(\nabla \times \overline{\mathrm{H}})=0 \\
\text { B-1 } \\
\text { B-2 }
\end{gathered}
$$




\subsection{The Universal Integration of Maxwell's Theory of Electrodynamics:}

The universal equation (19) for any arbitrary electromagnetic field configuration can be written in the form:

$$
\begin{gathered}
-\frac{1}{c^{2}} \frac{\partial(\overline{\mathrm{E}} \times \overline{\mathrm{H}})}{\partial t}+\varepsilon_{0} \overline{\mathrm{E}}(\nabla \cdot \overline{\mathrm{E}})-\varepsilon_{0} \overline{\mathrm{E}} \times(\nabla \times \overline{\mathrm{E}})+\mu_{0} \overline{\mathrm{H}}(\nabla \cdot \overline{\mathrm{H}})-\mu_{0} \overline{\mathrm{H}} \times(\nabla \times \overline{\mathrm{H}})=0 \\
-\varepsilon_{0} \mu_{0}\left(\overline{\mathrm{E}} \times \frac{\partial(\overline{\mathrm{H}})}{\partial t}+\overline{\mathrm{H}} \times \frac{\partial(\overline{\mathrm{E}})}{\partial t}\right)+\varepsilon_{0} \overline{\mathrm{E}}(\nabla \cdot \overline{\mathrm{E}})-\varepsilon_{0} \overline{\mathrm{E}} \times(\nabla \times \overline{\mathrm{E}})+\mu_{0} \overline{\mathrm{H}}(\nabla \cdot \overline{\mathrm{H}})-\mu_{0} \overline{\mathrm{H}} \times(\nabla \times \overline{\mathrm{H}})=0 \\
-\left(\varepsilon_{0} \overline{\mathrm{E}} \times \frac{\partial(\overline{\mathrm{B}})}{\partial t}+\mu_{0} \overline{\mathrm{H}} \times \frac{\partial(\overline{\mathrm{D}})}{\partial t}\right)+\overline{\mathrm{E}}(\nabla \cdot \overline{\mathrm{D}})-\varepsilon_{0} \overline{\mathrm{E}} \times(\nabla \times \overline{\mathrm{E}})+\overline{\mathrm{H}}(\nabla \cdot \overline{\mathrm{B}})-\mu_{0} \overline{\mathrm{H}} \times(\nabla \times \overline{\mathrm{H}})=0 \\
\mathrm{M}-3 \quad \mathrm{M}-4 \quad \mathrm{M}-3 \quad \mathrm{M}-2 \quad \mathrm{M}-4
\end{gathered}
$$

The Maxwell Equations are presented in (21):

$$
\begin{array}{llll}
\nabla \cdot \bar{D}=\rho & (\mathrm{M}-1) & \nabla \times \overline{\mathrm{E}}=-\frac{\partial \mathrm{B}}{\partial t}(\mathrm{M}-3) \\
\nabla \cdot \overline{\mathrm{B}}=0 & (\mathrm{M}-2) & \nabla \times \overline{\mathrm{H}}=\frac{\partial \mathrm{D}}{\partial t}(\mathrm{M}-4)
\end{array}
$$

In vacuum in the absence of any charge density, it follows from (26) that all the solutions for the Maxwell's Equations are also solutions for the separate parts of the Universal Equation (25) for the Electromagnetic field.

Universal Equation for the Electromagnetic Field.

$$
\begin{aligned}
& -\left(\varepsilon_{0} \overline{\mathrm{E}} \times \frac{\partial(\overline{\mathrm{B}})}{\partial t}+\mu_{0} \overline{\mathrm{H}} \times \frac{\partial(\overline{\mathrm{D}})}{\partial t}\right)+\overline{\mathrm{E}}(\nabla \cdot \overline{\mathrm{D}})-\varepsilon_{0} \overline{\mathrm{E}} \times(\nabla \times \overline{\mathrm{E}})+\overline{\mathrm{H}}(\nabla \cdot \overline{\mathrm{B}})-\mu_{0} \overline{\mathrm{H}} \times(\nabla \times \overline{\mathrm{H}})=0 \\
& \begin{array}{llllll}
\text { M-3 } & \text { M-4 } & \text { M-1 } & \text { M-3 } & \text { M-2 } & \text { M-4 }
\end{array} \\
& 4 \text { Maxwell's Equations } \\
& \nabla \cdot \bar{D}=\rho \quad(\mathrm{M}-1) \quad \nabla \times \overline{\mathrm{E}}=-\frac{\partial \mathrm{B}}{\partial t}(\mathrm{M}-3) \\
& \nabla \cdot \overline{\mathrm{B}}=0 \quad(\mathrm{M}-2) \quad \nabla \times \overline{\mathrm{H}}=\frac{\partial \mathrm{D}}{\partial t}(\mathrm{M}-4)
\end{aligned}
$$


Comparing the 4 Maxwell Equations (26) with the Universal Equation (24) we conclude that the 4 Maxwell equations

show only the 4 parts of the Universal Dynamic Equilibrium in 4 separate terms and the 4 Maxwell equations are missing the fundamental term for inertia. For that reason it is impossible to calculate the interaction between light and gravity with the 4 Maxwell equations. To find the interaction terms between light and gravity the inertia term (B-1 in 24) is necessary. 


\section{The Fundamental Discussion between Newton and Bohr}

The physical concept of quantum mechanical probability waves has been created during the famous $19275^{\text {th }}$ Solvay Conference. During that period there were several circumstances which came just together and made it possible to create a unique idea of "Material Waves" (Solutions of Schödinger's wave equation) being complex (partly real and partly imaginary) and describing the probability of the appearance of a physical object (elementary particle) generally indicated as "Quantum Mechanical Probability Waves".

\subsection{Newton's approach to the Fundamental Concept of Probability based on Newtonian Physics.}

Newton's second law can be interpreted as the law of "Conservation of Energy". For an Electromagnetic Field the law of conservation of Energy has been expressed as:

$$
\nabla \cdot \bar{S}+\frac{\partial w}{\partial t}=0
$$

From the equation for the "Conservation of Electromagnetic Energy" the Relativistic Quantum Mechanical "Dirac" equation will be derived which can be considered to be the relativistic version of the Quantum Mechanical Schrodinger wave equation.

\subsection{The fundamental concept of Probability}

The idea of complex (probability) waves is directly related to the concept of confined (standing) waves. Characteristic for any standing wave is the fact that the velocity and the pressure (electric field and magnetic field) are always shifted 14 
over 90 degrees. The same principle does exist for the standing (confined) electromagnetic waves,

For that reason every confined (standing) Electromagnetic wave can be described by a complex sum vector $\bar{\phi}$ of the Electric Field Vector $\overline{\mathrm{E}}$ and the Magnetic Field Vector $\overline{\mathrm{B}}(\overline{\mathrm{E}}$ has 90 degrees phase shift compared to $\overline{\mathrm{B}}$ ).

The vector functions $\bar{\phi}$ and the complex conjugated vector function $\bar{\phi}^{*}$ will be written as:

$$
\bar{\phi}=\frac{1}{\sqrt{2 \mu}}\left(\overline{\mathrm{B}}+\mathrm{i} \frac{\overline{\mathrm{E}}}{\mathrm{c}}\right)
$$

$\overline{\mathrm{B}}$ equals the magnetic induction, $\overline{\mathrm{E}}$ the electric field intensity $(\overline{\mathrm{E}}$ has +90 degrees phase shift compared to $\overline{\mathrm{B}})$ and $\mathrm{c}$ the speed of light.

The complex conjugated vector function equals:

$$
\overline{\phi^{*}}=\frac{1}{\sqrt{2 \mu}}\left(\overline{\mathrm{B}}-\mathrm{i} \frac{\overline{\mathrm{E}}}{\mathrm{c}}\right)
$$

The dot product equals the electromagnetic energy density w:

$$
\bar{\phi} \cdot \overline{\phi^{*}}=\frac{1}{2 \mu}\left(\overline{\mathrm{B}}+\mathrm{i} \frac{\overline{\mathrm{E}}}{\mathrm{c}}\right) \cdot\left(\overline{\mathrm{B}}-\mathrm{i} \frac{\overline{\mathrm{E}}}{\mathrm{c}}\right)=\frac{1}{2} \mu \mathrm{H}^{2}+\frac{1}{2} \varepsilon \mathrm{E}^{2}=\mathrm{W}
$$

Using Einstein's equation $\mathrm{W}=\mathrm{m} \mathrm{c}^{2}$, the dot product equals the electromagnetic mass density $w$ 
$\bar{\phi} \cdot \overline{\phi^{*}} \frac{1}{\mathrm{c}^{2}}=\frac{\varepsilon}{2}\left(\overline{\mathrm{B}}+\mathrm{i} \frac{\overline{\mathrm{E}}}{\mathrm{c}}\right) \cdot\left(\overline{\mathrm{B}}-\mathrm{i} \frac{\overline{\mathrm{E}}}{\mathrm{c}}\right)=\frac{1}{2} \varepsilon \mu^{2} \mathrm{H}^{2}+\frac{1}{2} \varepsilon^{2} \mathrm{E}^{2}=\rho\left[\mathrm{kg} / \mathrm{m}^{3}\right]$

The cross product is proportional to the Poynting vector (Ref. 3, page 202, equation 15).

$\bar{\phi} \times \overline{\phi^{*}}=\frac{1}{2 \mu}\left(\overline{\mathrm{B}}+\mathrm{i} \frac{\overline{\mathrm{E}}}{c}\right) \times\left(\overline{\mathrm{B}}-\mathrm{i} \frac{\overline{\mathrm{E}}}{c}\right)=\mathrm{i} \sqrt{\varepsilon \mu} \overline{\mathrm{E}} \times \overline{\mathrm{H}}=\mathrm{i} \sqrt{\varepsilon \mu} \overline{\mathrm{S}}$

Newton's second law of motion has been described in 3 spatial dimensions, resulting in the fundamental equation for the electromagnetic field.

$$
\begin{gathered}
\text { 3-Dimensional Space Domain } \\
\left(\begin{array}{c}
\mathrm{x}_{3} \\
\mathrm{x}_{2} \\
\mathrm{x}_{1}
\end{array}\right)+\mu_{0} \overline{\mathrm{H}}(\nabla \cdot \overline{\mathrm{H}})-\mu_{0} \overline{\mathrm{H}} \times(\nabla \times \overline{\mathrm{H}})+\frac{1}{2}\left(\varepsilon^{2} \mu(\overline{\mathrm{E}} \cdot \overline{\mathrm{E}})+\varepsilon \mu^{2}(\overline{\mathrm{H}} \cdot \overline{\mathrm{H}})\right) \overline{\mathrm{g}}=\overline{0} \\
c^{2}-\frac{1}{\partial t}+\varepsilon_{0} \overline{\mathrm{E}}(\nabla . \overline{\mathrm{E}})-\varepsilon_{0} \overline{\mathrm{E}} \times(\nabla \times \overline{\mathrm{E}})+
\end{gathered}
$$

The formal mathematical way to describe the force density results from the 4-dimensional divergence of the 4dimensional energy momentum tensor, resulting in a 4dimensional Force vector. Dividing the 4-dimensional Force vector by the Volume results in the 4-dimensional force density vector.

The 4-dimensional Electromagnetic Vector Potential has been defined by: 


$$
\varphi^{-4}=\left(\begin{array}{c}
\varphi_{4} \\
\varphi_{3} \\
\varphi_{2} \\
\varphi_{1}
\end{array}\right) \stackrel{\text { CartesianCoordinateSystem }}{\longrightarrow}\left(\begin{array}{c}
\varphi_{\mathrm{t}} \\
\varphi_{\mathrm{z}} \\
\varphi_{\mathrm{y}} \\
\varphi_{\mathrm{x}}
\end{array}\right)
$$

In which the term $\varphi_{\mathrm{a}}$ represents the 4-dimensional electromagnetic vector potential in the "a" direction while the indice "a" varies from 1 to 4 . In a cartesian coordinate system the indices are chosen varying from the $\mathrm{x}, \mathrm{y}, \mathrm{z}$ and $\mathrm{t}$ direction. In which the indice " $\mathrm{t}$ " represents the time direction which has been considered to be the $4^{\text {th }}$ dimension. The 4dimensional Electromagnetic "Maxwell Tensor" has been defined by:

$$
\mathrm{F}_{a b}=\partial_{b} \varphi_{a}-\partial_{a} \varphi_{b}
$$

Where the indices "a" and "b" vary from 1 to 4 .

The 4-dimensional Electromagnetic "Energy Momentum Tensor" has been defined by:

$$
T^{a b}=\frac{1}{\mu_{0}}\left[F_{a c} F^{c b}+\frac{1}{4} \delta_{a b} F_{c d} F^{c d}\right]
$$

The 4-dimensional divergence of the 4-dimensional Energy Momentum Tensor equals the 4-dimensional Force Density 4-vector $f^{a}$ : 
$f^{a}=\partial_{b} \mathrm{~T}^{a b}$

Substituting the electromagnetic values for the electric field intensity "E" and the magnetic field intensity " $\mathrm{H}$ " in (71) results in the 4-dimensional representation of Newton's second law of motion:

$$
\begin{gathered}
\begin{array}{c}
\text { Energy-Time Domain } \\
\text { B-7 }
\end{array} \\
\left(\mathrm{f}_{4}\right) \quad \nabla \cdot(\overline{\mathrm{E}} \times \overline{\mathrm{H}})+\frac{1}{2} \frac{\partial\left(\varepsilon_{0}(\overline{\mathrm{E}} \cdot \overline{\mathrm{E}})+\mu_{0}(\overline{\mathrm{H}} \cdot \overline{\mathrm{H}})\right)}{\partial t}=0 \\
\left(\begin{array}{c}
\mathrm{f}_{3} \\
\mathrm{f}_{2} \\
\mathrm{f}_{1}
\end{array}\right) \quad \begin{array}{c}
\text { 3-Dimensional Space Domain } \\
c^{2} \frac{\partial(\overline{\mathrm{E}} \times \overline{\mathrm{H}})}{\partial t}+\varepsilon_{0} \overline{\mathrm{E}}(\nabla \cdot \overline{\mathrm{E}})-\varepsilon_{0} \overline{\mathrm{E}} \times(\nabla \times \overline{\mathrm{E}})+ \\
+\mu_{0} \overline{\mathrm{H}}(\nabla \cdot \overline{\mathrm{H}})-\mu_{0} \overline{\mathrm{H}} \times(\nabla \times \overline{\mathrm{H}})=\overline{0}
\end{array}
\end{gathered}
$$

In which $f_{1}, f_{2}, f_{3}$, represent the force densities in the 3 spatial dimensions and $\mathrm{f}_{4}$ represent the force density (energy flow) in the time dimension ( $4^{\text {th }}$ dimension). Equation (42) can be written as:

The $4^{\text {th }}$ term in equation (42) can be written in the terms of the Poynting vector "S" and the energy density " $\mathrm{w}$ " representing the electromagnetic law for the conservation of energy (Newton's second law of motion). 


$$
\begin{aligned}
& \text { Energy-Time Domain } \\
& \text { Inner Energy } \\
& \text { B-7 } \\
& \left(\mathrm{f}_{4}\right) \\
& \nabla \cdot \overline{\mathrm{S}}+\frac{\partial \mathrm{W}}{\partial t} \quad=0 \\
& \text { B-1 B-2 B-3 }
\end{aligned}
$$

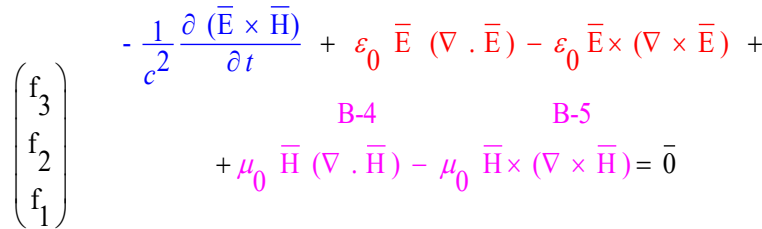




\subsection{The 4-Dimensional Relativistic Quantum Mechanical Dirac Equation}

Substituting (27) and (28) in Equation (35.1) results in The 4Dimensional Equilibrium Equation (36):

$$
\begin{gathered}
\left.\left(\mathrm{x}_{4}\right)-\frac{i}{\sqrt{\varepsilon_{0} \mu_{0}}} \nabla \cdot \bar{\phi} \times \bar{\phi}\right)=-\frac{\partial \bar{\phi} \cdot \bar{\phi}^{*}}{\partial t} \\
\left(\begin{array}{l}
\mathrm{x}_{3} \\
\mathrm{x}_{2} \\
\mathrm{x}_{1}
\end{array}\right) \begin{array}{c}
-\frac{1}{c^{2}} \frac{\partial(\overline{\mathrm{E}} \times \overline{\mathrm{H}})}{\partial t}+\varepsilon_{0} \overline{\mathrm{E}}(\nabla \cdot \overline{\mathrm{E}})-\varepsilon_{0} \overline{\mathrm{E}} \times(\nabla \times \overline{\mathrm{E}})+ \\
+\mu_{0} \overline{\mathrm{H}}(\nabla \cdot \overline{\mathrm{H}})-\mu_{0} \overline{\mathrm{H}} \times(\nabla \times \overline{\mathrm{H}})=\overline{0}
\end{array}
\end{gathered}
$$

To transform the electromagnetic vector wave function $\bar{\phi}$ into a scalar (spinor or one-dimensional matrix representation), the Pauli spin matrices $\sigma$ and the following matrices (Ref. 3 page 213, equation 99) are introduced:

$$
\bar{\alpha}=\left[\begin{array}{ll}
0 & \sigma \\
\sigma & 0
\end{array}\right] \quad \text { and } \quad \bar{\beta}=\left[\begin{array}{cc}
\delta_{a b} & 0 \\
0 & -\delta_{a b}
\end{array}\right]
$$

Then equation (44) can be written as the 4-Dimensional Hyperspace Equilibrium Dirac Equation: 


$$
\begin{gathered}
\left(\mathrm{x}_{4}\right) \quad\left(\frac{\mathrm{imc}}{h} \bar{\beta}+\bar{\alpha} \cdot \nabla\right) \psi=-\frac{1}{c} \frac{\partial \psi}{\partial t} \\
\left(\begin{array}{l}
\mathrm{x}_{3} \\
\mathrm{x}_{2} \\
\mathrm{x}_{1}
\end{array}\right) \quad \begin{array}{c}
-\frac{1}{c^{2}} \frac{\partial(\overline{\mathrm{E}} \times \overline{\mathrm{H}})}{\partial t}+\varepsilon_{0} \overline{\mathrm{E}}(\nabla \cdot \overline{\mathrm{E}})-\varepsilon_{0} \overline{\mathrm{E}} \times(\nabla \times \overline{\mathrm{E}})+ \\
+\mu_{0} \overline{\mathrm{H}}(\nabla \cdot \overline{\mathrm{H}})-\mu_{0} \overline{\mathrm{H}} \times(\nabla \times \overline{\mathrm{H}})=\overline{0}
\end{array}
\end{gathered}
$$

The fourth term $\left(\mathrm{x}_{4}\right)$ equals the relativistic Dirac equation (38.1) which equals equation (102) page 213 in Ref.3.

Equation (38.1) represents the relativistic quantum mechanical Dirac Equation where $\psi$ represents the quantum mechanical probability wave function. The mathematical evidence for the equivalent for (38.1) has been published in 1995 in the article: "A Continuous Model of Matter based on AEONs". Equation (1) page 201 to Equation (102) page 213. (Doi: 10.31219/osf.io/ra7ng)

The Electromagnetic Law for the conservation of Energy (35.1) and the Relativistic Dirac Equation (38.1) are identical but written in a different form.

The law of conservation of Electromagnetic Energy can be written in an electromagnetic form (39.1) or in an identical way in a quantum mechanical form (39.2): 


\section{Energy-Time Domain \\ Inner Energy}

B-7

$\left(\mathrm{f}_{4}\right)$

$$
\nabla \cdot(\bar{\phi} \times \bar{\phi})=-\frac{i}{c} \frac{\partial \bar{\phi} \cdot \bar{\phi}^{*}}{\partial t}
$$

$$
\left(\mathrm{x}_{4}\right) \quad\left(\frac{\mathrm{imc}}{h} \bar{\beta}+\bar{\alpha} \cdot \nabla\right) \psi=-\frac{1}{c} \frac{\partial \psi}{\partial t}
$$

The weakness in the Quantum Mechanical Relativistic Dirac Equation (39.2) is that the Dirac Equation is a 1-dimensional equation which will never be able to describe the 4dimensional real physical world. While 39.1 represents a ElectromagneticVector Equation.

From the equations (27) and (28) follows the 4-Dimensional Vector-Dirac equation (40). This equation is a 4-dimensional vector equation and is coherent with the 4-dimensional physical reality.

$$
\begin{gathered}
\left(\begin{array}{l}
x_{4} \\
4
\end{array}\right)=(\bar{\phi} \times \bar{\phi})=-\frac{i}{c} \frac{\partial \bar{\phi} \cdot \bar{\phi}^{*}}{\partial t} \\
\left(\begin{array}{l}
x_{3} \\
x_{2} \\
x_{1}
\end{array}\right) \frac{i \frac{\partial(\bar{\phi} \times \bar{\phi})}{c}}{c}-\left(\bar{\phi} \times\left(\nabla \times \overline{\phi^{*}}\right)+\overline{\phi^{*}} \times(\nabla \times \bar{\phi})\right)+\left(\bar{\phi}\left(\nabla \cdot \overline{\phi^{*}}\right)+\overline{\phi^{*}}(\nabla \cdot \bar{\phi})\right)=0
\end{gathered}
$$

In which the Quantum Mechanical Complex Probability Vector Function $\bar{\phi}$ and the complex conjugated vector function $\overline{\phi^{*}}$ equals: 


$$
\begin{aligned}
& \bar{\phi}=\overline{\mathrm{B}}+\frac{i}{c} \overline{\mathrm{E}}=\mu \overline{\mathrm{H}}+\frac{i}{c} \overline{\mathrm{E}} \\
& \overline{\phi^{*}}=\overline{\mathrm{B}}-\frac{i}{c} \overline{\mathrm{E}}=\mu \overline{\mathrm{H}}-\frac{i}{c} \overline{\mathrm{E}}
\end{aligned}
$$

The 4-Dimensional Dirac equation represents the "Newtonian Perfect Equilibrium" in the 4-Dimensional Space-Time Continuum en has been represented by 4 separate equations. The first one represents the well-known relativistic quantum mechanical Dirac Equation in the Time-Energy domain X4. The 3 quantum mechanical equations in the space-momentum domain represents the "Newtonian Perfect Equilibrium" for the force densities in the domains $\left(\mathrm{x}_{1}, \mathrm{x}_{2}, \mathrm{x}_{3}\right)$

$$
\begin{aligned}
& \left(\mathrm{x}_{4}\right) \quad \nabla \cdot(\bar{\phi} \times \bar{\phi})=-\frac{i}{c} \frac{\partial \bar{\phi} \cdot \bar{\phi}^{*}}{\partial t} \\
& \left(\begin{array}{l}
\mathrm{x}_{3} \\
\mathrm{x}_{2} \\
\mathrm{x}_{1}
\end{array}\right) \frac{i}{c} \frac{\partial\left(\bar{\phi} \times \overline{\phi^{*}}\right)}{\partial t}-\left(\bar{\phi} \times\left(\nabla \times \overline{\phi^{*}}\right)+\overline{\phi^{*}} \times(\nabla \times \bar{\phi})\right)+\left(\bar{\phi}\left(\nabla \cdot \overline{\phi^{*}}\right)+\overline{\phi^{*}}(\nabla \cdot \bar{\phi})\right)=0 \\
& \text { Newton } \\
& \text { Lorentz } \\
& \text { Coulomb } \\
& \text { Newtonian Perfect Equilibrium } \\
& \frac{1}{\mathrm{c}^{2}} \bar{\phi} \cdot \overline{\phi^{*}}=\rho\left[\mathrm{kg} / \mathrm{m}^{3}\right]
\end{aligned}
$$

These results lead to the conclusion that the results of the experiments, published in 2021 "Operational Resource Theory of Imaginarity " in "Physical Review Letters" present strong evidence for the existence at subatomic level of the electromagnetic GEONs and the correctness of Wheeler's theory (Equation (50) and (51). 
To define the Fundamental Equation for the Interaction between Gravity and Light, an extra term (B-6) has been introduced in equation (29). The term B-6 represents the force density of the gravitational field acting on the electromagnetic mass density.

$$
\mathrm{F}_{\text {GRAVITY }}=\mathrm{m} \overline{\mathrm{g}}[\mathrm{N}]
$$

Dividing both terms by the Volume V:

$\frac{\mathrm{F}_{\text {GRAVITY }}}{\mathrm{V}}=\frac{\mathrm{m}}{\mathrm{V}} \overline{\mathrm{g}}\left[\mathrm{N} / \mathrm{m}^{3}\right]$

Results in the force density:

$$
\mathrm{f}_{\text {GRAVITY }}=\rho \overline{\mathrm{g}}\left[\mathrm{N} / \mathrm{m}^{3}\right]
$$

The specific mass " $\rho$ " of a beam of light follows from Einstein's equation:

$$
\mathrm{W}=\mathrm{m} \mathrm{c}^{2}
$$

Divinding both terms by the Volume V results in:

$$
\frac{\mathrm{W}}{\mathrm{V}}=\frac{\mathrm{m}}{\mathrm{V}} \mathrm{c}^{2}
$$

which represents the energy density "w" and the specific mass " $\rho$ " of the electromagnetic radiation:

$\mathrm{w}=\rho \mathrm{c}^{2}$

which results for an expression of the specific mass $\rho$ : $\rho=\frac{1}{\mathrm{c}^{2}} \mathrm{w}=\varepsilon \mu \mathrm{w}$

The energy density "w" follows from the electric and the magnetic field intensities: 


$$
\begin{aligned}
& \mathrm{w}=\frac{1}{2} \varepsilon \mathrm{E}^{2}+\frac{1}{2} \mu \mathrm{H}^{2} \\
& \mathrm{w}=\frac{1}{2}\left(\varepsilon \mathrm{E}^{2}+\mu \mathrm{H}^{2}\right)=\frac{1}{2}(\varepsilon(\overline{\mathrm{E}} \cdot \overline{\mathrm{E}})+\mu(\overline{\mathrm{H}} \cdot \overline{\mathrm{H}}))
\end{aligned}
$$

Substituting equation (45) in equation (29) results in the gravitational force density f $f_{\text {GRAVITY }}$ acting on an arbitrary electromagnetic field configuration (a beam of light) with mass density $\rho$.

$$
\begin{aligned}
& \mathrm{f}_{\text {GRAVITY }}=\rho \overline{\mathrm{g}} \\
& \mathrm{f}_{\text {GRAVITY }}=\rho \overline{\mathrm{g}}=\varepsilon \mu \mathrm{w} \overline{\mathrm{g}}=\frac{1}{2}\left(\varepsilon^{2} \mu(\overline{\mathrm{E}} \cdot \overline{\mathrm{E}})+\varepsilon \mu^{2}(\overline{\mathrm{H}} \cdot \overline{\mathrm{H}})\right) \overline{\mathrm{g}}
\end{aligned}
$$

Substituting equation (46) in equation (29) results in the fundamental equation describing the ElectromagneticGravitational interaction for any arbitrary electromagnetic field configuration (a beam of light):

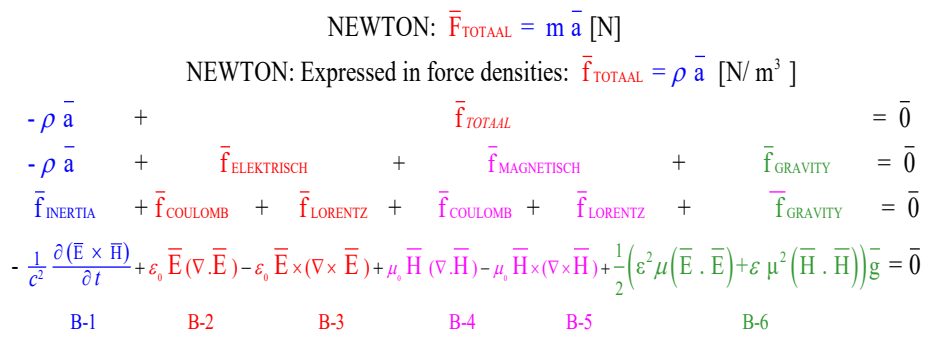

Term B-1 represents the inertia term of the electromagnetic radiation. Term B-4 is the magnetic representation of the (electric) Coulomb's Force B-2 and Term B-3 is the electric representation of the (magnetic) Lorentz Force B-5. Term B6 represents the Electromagnetic-Gravitational interaction of a gravitational field with field acceleration $\bar{g}$ acting on an 
arbitrary electromagnetic field configuration ( a beam of light) with specific mass $\rho$.

The universal equation for the electromagnetic field (free electromagnetic waves and confined electromagnetic fields) within a gravitational field with gravity field intensity $\overline{\mathrm{g}}$ has been presented in (48) and expresses the perfect equilibrium between the inertia forces (B-1), the electric forces (B-2 and B-3), the magnetic forces (B-4 and B-5) and the gravitational force (B-6) in any arbitrary electromagnetic field configuration.

$$
\begin{gathered}
-\frac{1}{c^{2}} \frac{\partial(\overline{\mathrm{E}} \times \overline{\mathrm{H}})}{\partial t}+\varepsilon_{0} \overline{\mathrm{E}}(\nabla \cdot \overline{\mathrm{E}})-\varepsilon_{0} \overline{\mathrm{E}} \times(\nabla \times \overline{\mathrm{E}})+\mu_{0} \overline{\mathrm{H}}(\nabla \cdot \overline{\mathrm{H}})- \\
\text { B-1 } \quad \text { B-2 } \quad \text { B-3 }-4 \\
-\mu_{0} \overline{\mathrm{H}} \times(\nabla \times \overline{\mathrm{H}})+\frac{1}{2}\left(\varepsilon^{2} \mu(\overline{\mathrm{E}} \cdot \overline{\mathrm{E}})+\varepsilon \mu^{2}(\overline{\mathrm{H}} \cdot \overline{\mathrm{H}})\right) \overline{\mathrm{g}}=\overline{0}
\end{gathered}
$$

B-5

B-6 


\subsection{The Gravitational-Electromagnetic Confinement of GEONs.}

The physicist "John Archibald Wheeler", and well-known friend of Albert Einstein, published his theory about Gravitational Electromagnetic Entities (GEONs) in 1955 in Physical Review Journals: "GEONs" (Ref. 1). John Archibald Wheeler was the first one in physics who understood that:

$$
\begin{aligned}
& \text { Light } \hat{=} \text { Electromagnetic Radiation } \hat{=} \text { Energy } \hat{=} \\
& \hat{=} \text { Mass }\left(E=m c^{2}\right) \hat{=} \text { Gravity } \hat{=} \text { Gravitational Field }
\end{aligned}
$$

Wheeler's conclusion was that every beam of light creates simultaneously a gravitational field, besides the original electromagnetic field, proportional to the electromagnetic energy of the beam of light.

Like a Black Hole can capture a beam of light, it is possible that a beam of light confines itself due to its own gravitational field forming a Gravitational Electromagnetic Entity (GEON). The final results of Wheeler's research were not what he expected. He found only GEONs by toroidal "Gravitational-Electromagnetic Confinement" with the dimensions of a star constellation and a stable lifetime of a few milliseconds.

"Quantum Light Theory" presents a new "GravitationalElectromagnetic Equation" (Ref. [7], [8] and [9]) describing Electromagnetic Field Configurations which are simultaneously the Mathematical Solutions for the Quantum Mechanical "Schrodinger Wave Equation" and more exactly the Mathematical Solutions for the "Relativistic Quantum Mechanical Dirac Equation". The Mathematical Solutions for 
the "Gravitational-Electromagnetic Equation" carry Mass, Electric Charge and Magnetic Spin at discrete values.

When a beam of light is approaching a strong gravitational field in the direction of the gravitational field, generated by a Black Hole, the confinement has been called a Longitudinal Black Hole. The direction of propagation of the beam of light is in the same direction (or in the opposite direction) of the gravitational field. According the first term in (33), the beam of light will be accelerated or decelerated. However, the speed of light is a universal constant and for that reason the speed of light cannot increase or decrease. Instead the intensity of the electromagnetic radiation will increase when the beam of light approaches (propagates in the opposite direction as the direction of the gravitational field) the Black Hole. And the intensity of the electromagnetic radiation will decrease when the beam of light leaves (propagates in the same direction as the direction of the gravitational field) the Black Hole.

The Gravitational-Electromagnetic Confinement for the elementary structure beyond the "superstring" / "Black Hole" is presented in equation (49).

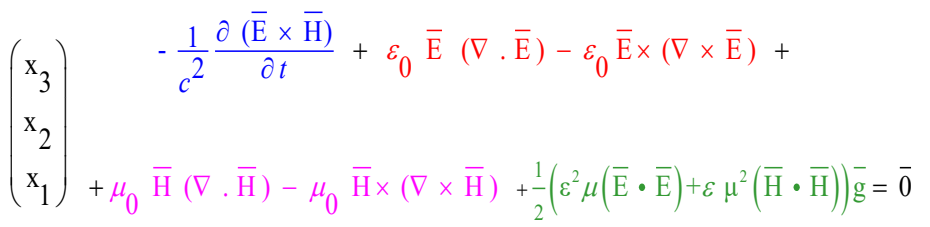


In which $\bar{g}$ represents the gravitational acceleration acting on the electromagnetic mass density of the confined electromagnetic radiation.

A possible solution for equation (49) describing an Gravitational Electromagnetic Confinement (GEON) within a radial gravitational field with acceleration $\overline{\mathrm{g}}$ has been represented in (50).

$$
\begin{aligned}
& \left(\begin{array}{l}
e_{r} \\
e_{\theta} \\
e_{\varphi}
\end{array}\right)=\left(\begin{array}{c}
0 \\
\mathrm{f}(\mathrm{r}) \operatorname{Sin}(\omega \mathrm{t}) \\
-\mathrm{f}(\mathrm{r}) \operatorname{Cos}(\omega \mathrm{t})
\end{array}\right) \quad\left(\begin{array}{l}
m_{r} \\
m_{\theta} \\
m_{\varphi}
\end{array}\right)=\left(\begin{array}{c}
0 \\
\mathrm{f}(\mathrm{r}) \operatorname{Cos}(\omega \mathrm{t}) \\
\mathrm{f}(\mathrm{r}) \operatorname{Sin}(\omega \mathrm{t})
\end{array}\right) \quad \bar{g}=\left(\begin{array}{c}
\frac{G_{1}}{4 \pi \mathrm{r}^{2}} \\
0 \\
0
\end{array}\right) \\
& \mathrm{W}_{\mathrm{em}}=\left(\frac{\mu_{0}}{2}(\overline{\mathrm{m}} \cdot \overline{\mathrm{m}})+\frac{\varepsilon_{0}}{2}(\overline{\mathrm{e}} \cdot \overline{\mathrm{e}})\right)=\varepsilon_{0} \mathrm{f}(r)^{2}
\end{aligned}
$$

In which the radial function $\mathrm{f}(\mathrm{r})$ equals:

$$
f[r]=K \mathrm{e}^{-\frac{-\frac{G 1 \varepsilon_{0} \mu_{0}}{r}+8 \pi \log [r]}{8 \pi}}
$$

The solution has been calculated according Newton's Shell Theorem. 
2.5 GEONs" with an electromagnetic mass: $\mathrm{e}_{\mathrm{mm}}=\mathbf{1 . 6 7 2 6}$ $\times 10^{-27}[\mathrm{~kg}]$ and the radius $=3 \times 10^{-58}[\mathrm{~m}]$

For an electromagnetic mass of the confinement: $\mathrm{e}_{\mathrm{mm}}=$ $1.6726 \times 10^{-27}[\mathrm{~kg}]$ (mass of proton), the radius of the confinement equals approximately $3 \times 10^{-58}[\mathrm{~m}]$. This is far beyond the order of Planck's Length,

The Plot graph of the Electric Field Intensity $f(r)$ of the confinement has been presented as a function of the radius in figure (2) and figure (3):

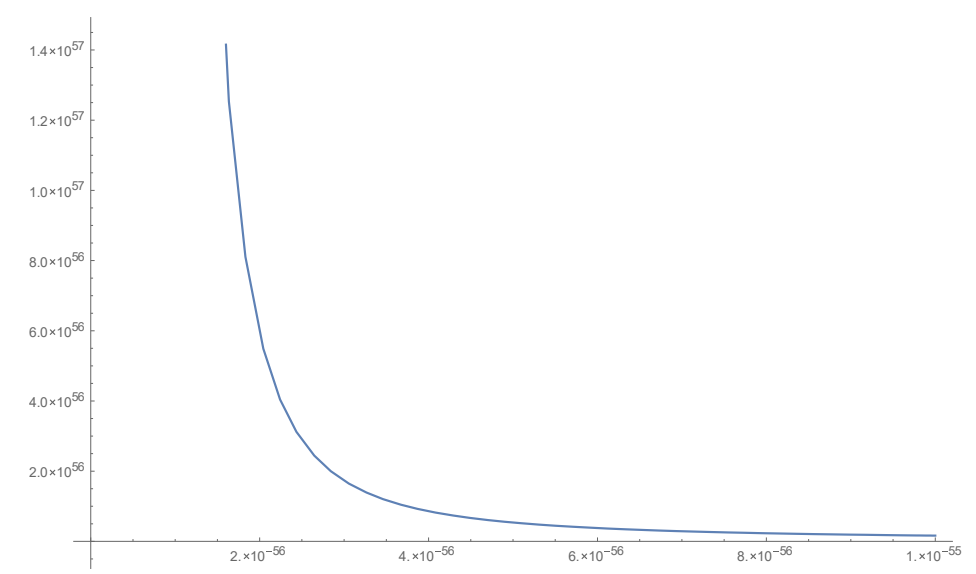

Figure 2 PlotGraph of the Electric Field Intensity $f(r)$ $[\mathrm{V} / \mathrm{m}]$ for the region $10^{-59}<\mathrm{r}<1^{-55}[\mathrm{~m}]$ in which the gravitational field acceleration has been chosen accordingly an electromagnetic mass of $1.6726 \times 10^{-27}$ [kg] located at the center of the confinement, according Newton's Shell Theorem. 


$$
\text { Plot }\left[\mathrm{e}^{-\frac{-\frac{G 1 \varepsilon_{0} \mu_{0}}{r}+8 \pi \log [r]}{8 \pi}},\left\{r, 10^{-59}, 10^{-57}\right\}\right]
$$

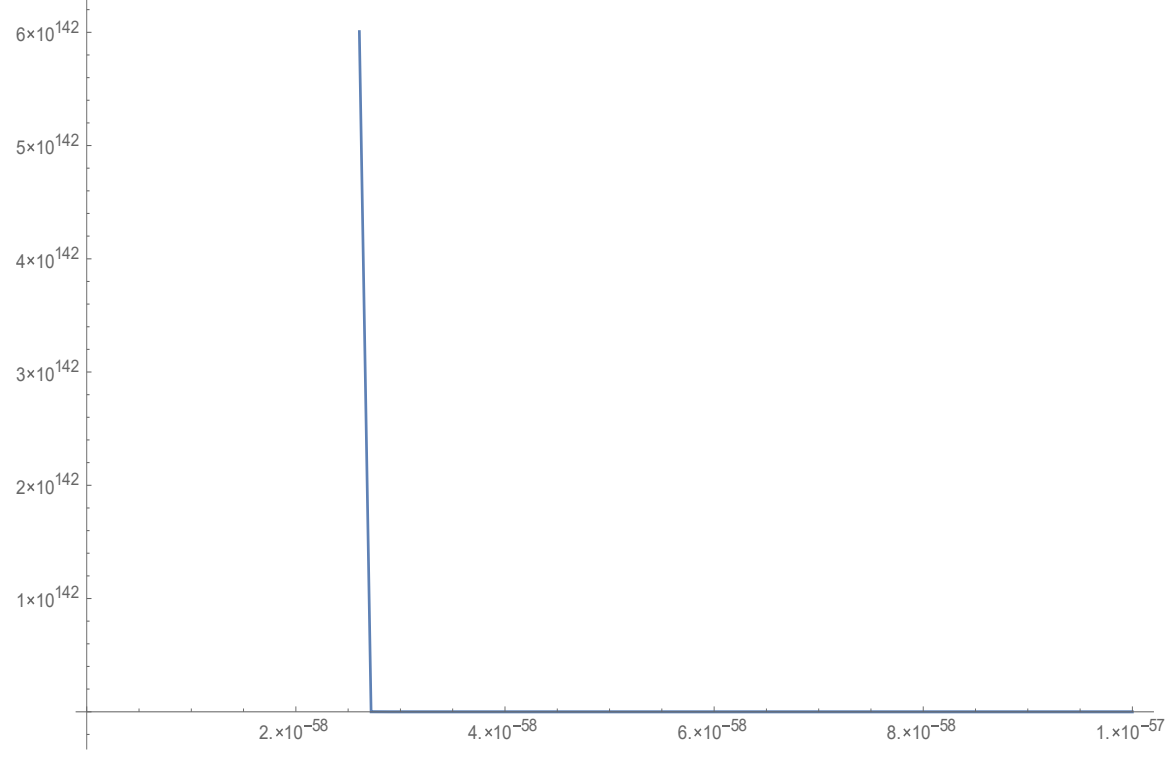

Figure 3 PlotGraph of the Electric Field Intensity $\mathbf{f}(\mathbf{r})$ $[\mathrm{V} / \mathrm{m}]$ for the region $10^{-59}<\mathbf{r}<1^{-57}[\mathrm{~m}]$ in which the gravitational field acceleration has been chosen accordingly an electromagnetic mass of $1.6726 \times 10^{-27}[\mathrm{~kg}]$ located at the center of the confinement, according Newton's Shell Theorem.

The fundamental question is: How it is possible to create confinements from "visible light" (with a wave length between $3.9 \times 10^{-7}[\mathrm{~m}]$ until $\left.7 \times 10^{-7}[\mathrm{~m}]\right)$ within dimensions smaller than Planck's Length? 
This is only possible when the wave length of the confined radiation is smaller than de dimensions of the confinement. This requires extreme high frequencies. The transformation in frequency from visible light into the extreme high frequency of the confinement is possible because of the Lorentz/ Doppler transformation during the collapse of the radiation when the confinement has been formed (implosion of visible light).

\subsection{The Electric Charge and the Magnetic Spin for Electromagnetic GEONs}

The following functions for Electromagnetic GEONs with the quantum variables $\left\{\mathrm{m}_{1}, \mathrm{n}_{1}, \mathrm{p}_{1}, \mathrm{q}_{1}\right\}$ have been chosen:

$$
\begin{aligned}
& f[r]=K \mathrm{e}^{-\frac{-\frac{G 1 \varepsilon_{0} \mu_{0}}{r}+8 \pi \log [r]}{8 \pi}} \\
& \mathrm{g}_{1}(\theta, \varphi, t)=\sin (t \omega)\left(\sin \left(\pi \theta \mathrm{m}_{1}\right) \sin \left(\mathrm{n}_{1} 2 \pi \varphi\right)+1\right) \\
& h(\theta, \varphi)=\sin \left(\pi \theta \mathrm{p}_{1}\right) \sin \left(\mathrm{q}_{1} 2 \pi \varphi\right)+1 \\
& \mathrm{~g}_{2}(\theta, \varphi, t)=\frac{\sec (t \omega) \sqrt{\cos (2 t \omega) \mathrm{g}_{1}(\theta, \varphi, t)^{2}-\mathrm{g}_{1}(\theta, \varphi, t)^{2}+2 h(\theta, \varphi)}}{\sqrt{2}} \\
& f_{1}[r, \theta, \varphi, t]=\mathrm{e}^{-\frac{-\frac{G 1 \varepsilon_{0} \mu_{0}}{r}+8 \pi \log [r]}{8 \pi}} K g_{1}[\theta, \varphi, t] \\
& f_{2}[r, \theta, \varphi, t]=\frac{\mathrm{e}^{-\frac{-\frac{G 1 \varepsilon_{0} \mu_{0}}{r}+8 \pi \log [r]}{8 \pi}} K \sqrt{-g_{1}[\theta, \varphi, t]^{2}+\cos [2 t \omega] g_{1}[\theta, \varphi, t]^{2}+2 h[\theta, \varphi]}}{\sqrt{2}}
\end{aligned}
$$




\subsection{GEON with quantum numbers: (Electric- and Magnetic Dipoles, Electric- and Magnetic Spin) $\left\{\mathbf{m}_{1}=\mathbf{0}, \mathbf{n}_{1}=\mathbf{0}, \mathbf{p}_{1}=\mathbf{0}, \mathbf{q}_{1}=\mathbf{0}\right\}$}

The divergence of the electric field intensity (electric charge density) equals:

$$
\begin{aligned}
& \nabla .\left(\begin{array}{l}
e_{r} \\
e_{\theta} \\
e_{\varphi}
\end{array}\right)=\frac{\sqrt{2} \mathrm{~K} 1 \cot (\theta) \sin ^{2}(t \omega) \sqrt{1-\sin ^{4}(t \omega)} \mathrm{e}^{\frac{G_{1} \varepsilon_{0} \mu_{0}}{8 \pi \mathrm{r}}}}{r^{2} \sqrt{2-2 \sin ^{4}(t \omega)}} \\
& \nabla .\left(\begin{array}{l}
e_{r} \\
e_{\theta} \\
e_{\varphi}
\end{array}\right)=\frac{\frac{1}{2} \mathrm{~K} 1 \cot (\theta) \mathrm{e}^{\frac{G_{1} \varepsilon_{0} \mu_{0}}{8 \pi \mathrm{r}}}}{r^{2}} \text { (averaged over 1 period of time) }
\end{aligned}
$$

The divergence of the magnetic field intensity (magnetic monopole) equals:

$$
\begin{aligned}
& \nabla .\left(\begin{array}{l}
m_{r} \\
m_{\theta} \\
m_{\varphi}
\end{array}\right)=\frac{\mathrm{K} 1 \sqrt{\varepsilon_{0}} \cot (\theta) \sqrt{2-2 \sin ^{4}(t \omega)} \mathrm{e}^{\frac{G_{1} \varepsilon_{0} \mu_{0}}{8 \pi \mathrm{r}}}}{\sqrt{2} \sqrt{\mu 0} r^{2}} \\
& \nabla .\left(\begin{array}{l}
m_{r} \\
m_{\theta} \\
m_{\varphi}
\end{array}\right)=\frac{\mathrm{K} 1 \sqrt{\varepsilon_{0}} \cot (\theta) \sqrt{\frac{3}{4}} \mathrm{e}^{\frac{G_{1} \varepsilon_{0} \mu_{0}}{8 \pi \mathrm{r}}}}{\sqrt{\mu 0} r^{2}} \text { (averaged over 1 period of time) }
\end{aligned}
$$

In which $\mathrm{K} 1$ is an arbitrary variable. Because of the $\operatorname{Cot}(\theta)$ function, the electric divergence as well as the magnetic divergence changes from sign when the angle $\theta$ varies between $0^{0}$ until $360^{\circ}$ forming electric dipoles (+ versus -) and magnetic dipoles ( $\mathrm{N}$ versus $\mathrm{S}$ ). 
2.8 GEON with quantum numbers: (Electric- and Magnetic Dipoles, Electric- and Magnetic Spin) $\left\{\mathbf{m}_{1}=\mathbf{1}, \mathbf{n}_{1}=\mathbf{0}, \mathbf{p}_{1}=\mathbf{0}, \mathbf{q}_{1}=\mathbf{0}\right\}$

3.26.2 Type 2 of "Sub Max Planck Length GravitationalElectromagnetic Confinements" (Electric- and Magnetic Dipoles, Electric- and Magnetic Spin) $\{\mathbf{m 1} 1=1, \mathbf{n 1}=0, \mathbf{p} 1=0$, q1 $=0$ \}

The divergence of the electric field intensity (electric charge density) equals:

$\nabla .\left(\begin{array}{l}e_{r} \\ e_{\theta} \\ e_{\varphi}\end{array}\right)=\frac{\sqrt{2} \mathrm{~K} 1 \cot (\theta) \sin ^{2}(t \omega) \sqrt{1-\sin ^{4}(t \omega)} \mathrm{e}^{\frac{G_{1} \varepsilon_{0} \mu_{0}}{8 \pi \mathrm{r}}}}{r^{2} \sqrt{2-2 \sin ^{4}(t \omega)}}$

$\nabla .\left(\begin{array}{l}e_{r} \\ e_{\theta} \\ e_{\varphi}\end{array}\right)=\frac{\frac{1}{2} \mathrm{~K} 1 \cot (\theta) \mathrm{e}^{\frac{G_{1} \varepsilon_{0} \mu_{0}}{8 \pi \mathrm{r}}}}{r^{2}}$ (averaged over 1 period of time)

The divergence of the magnetic field intensity (magnetic monopole) equals:

$\nabla \cdot\left(\begin{array}{l}m_{r} \\ m_{\theta} \\ m_{\varphi}\end{array}\right)=\frac{\mathrm{K} 1 \sqrt{\varepsilon_{0}} \cot (\theta) \sqrt{2-2 \sin ^{4}(t \omega)} \mathrm{e}^{\frac{G_{1} \varepsilon_{0} \mu_{0}}{8 \pi \mathrm{r}}}}{\sqrt{2} \sqrt{\mu_{0}} r^{2}}$

$\nabla .\left(\begin{array}{l}m_{r} \\ m_{\theta} \\ m_{\varphi}\end{array}\right)=\frac{\mathrm{K} 1 \sqrt{\frac{3}{4}} \sqrt{\varepsilon_{0}} \cot (\theta) \mathrm{e}^{\frac{G_{1} \varepsilon_{0} \mu_{0}}{8 \pi \mathrm{r}}}}{\sqrt{\mu_{0}} r^{2}}$ (averaged over 1 period of time) 
In which $\mathrm{K} 1$ is an arbitrary variable. Because of the $\operatorname{Cot}(\theta)$ function, the electric divergence as well as the magnetic divergence changes from sign when the angle $\theta$ varies between $0^{\circ}$ until $360^{\circ}$ forming electric dipoles (+ versus -) and magnetic dipoles ( $\mathrm{N}$ versus $\mathrm{S}$ ).

2.9 GEON with quantum numbers: (Electric- and Magnetic Dipoles, Electric- and Magnetic Spin) $\left\{m_{1}=0, n_{1}=0, p_{1}=1, q_{1}=0\right\}$

The divergence of the electric field intensity (electric charge density) equals:

$\nabla .\left(\begin{array}{l}e_{r} \\ e_{\theta} \\ e_{\varphi}\end{array}\right)=\frac{\mathrm{e}^{\frac{G_{1} \varepsilon_{0} \mu_{0}}{8 \pi \mathrm{r}}} K 1 \cot [\theta] \sin [t \omega]^{2}}{r^{2}}$

$\nabla \cdot\left(\begin{array}{l}e_{r} \\ e_{\theta} \\ e_{\varphi}\end{array}\right)=\frac{\mathrm{e}^{\frac{G_{1} \varepsilon_{\varepsilon_{0}} \mu_{0}}{8 \pi \mathrm{r}}} K 1 \cot [\theta]}{2 r^{2}}$ (averaged over 1 period of time) 
In which $\mathrm{K} 1$ is an arbitrary variable with a Positive (positive charge) or a Negative (negative charge) value.

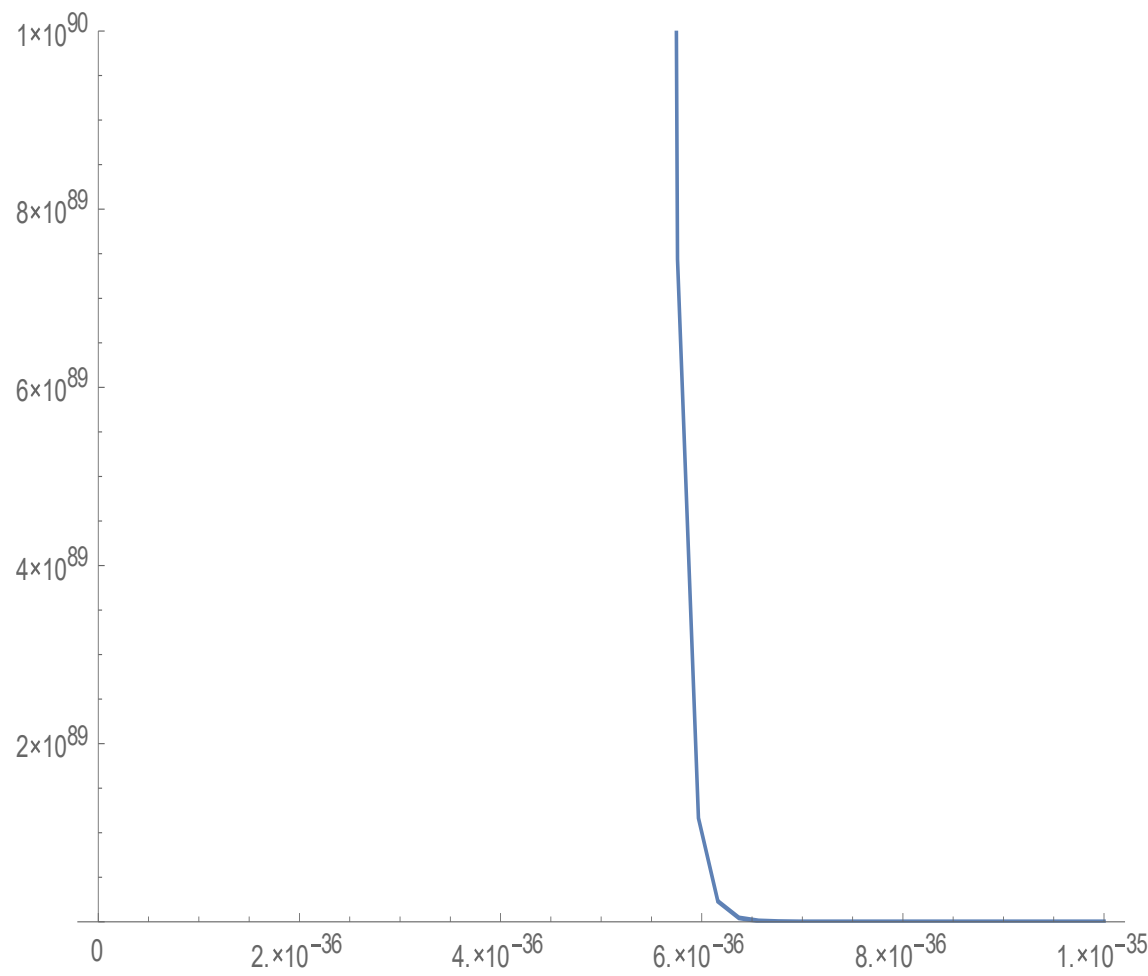

Figure 4 Equation (54). Averaged over 1 period of time. Electric Charge Density Plot in a range from $10^{-37}[\mathrm{~m}]$ until $10^{-35}[\mathrm{~m}]$. 
The divergence of the magnetic field intensity (magnetic monopole) equals:

$\nabla .\left(\begin{array}{l}m_{r} \\ m_{\theta} \\ m_{\varphi}\end{array}\right)=\frac{\mathrm{e}^{\frac{G_{1} \varepsilon_{0} \mu_{0}}{8 \pi \mathrm{r}}} K 1 \sqrt{\varepsilon_{0}} \cot [\theta] \sqrt{1-\sin [t \omega]^{4}}}{r^{2} \sqrt{\mu_{0}}}$
$\nabla .\left(\begin{array}{l}m_{r} \\ m_{\theta} \\ m_{\varphi}\end{array}\right)=\frac{\mathrm{e}^{\frac{G_{1} \varepsilon_{0} \mu_{0}}{8 \pi \mathrm{r}}} K 1 \sqrt{\varepsilon_{0}} \cot [\theta]}{2 r^{2} \sqrt{\mu_{0}}}$ (averaged over 1 period of time)

In which $\mathrm{K} 1$ is an arbitrary variable. Because of the $\operatorname{Cot}(\theta)$ function, the electric divergence as well as the magnetic divergence changes from sign when the angle $\theta$ varies between $0^{\circ}$ until $360^{\circ}$ forming electric dipoles (+ versus -) and magnetic dipoles ( $\mathrm{N}$ versus $\mathrm{S}$ ).

\subsection{Plank's Law in relation to Newtonian Physics.}

To describe the "proportionality between Energy and Frequency (Plank's Law) for confined Electromagnetic Waves, an example has been chosen of an electromagnetic wave (light) confined between two $100 \%$ reflecting mirrors.

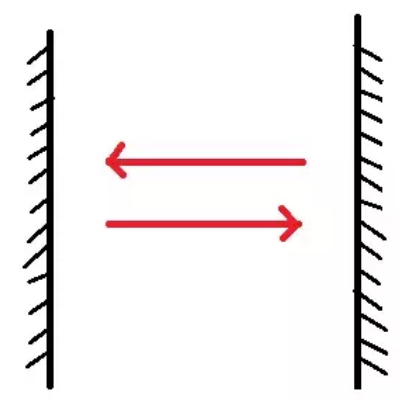

Figure 5 Confined Electromagnetic Radiation between two $100 \%$ reflecting mirrors 
Figure 2 represents confined electromagnetic radiation with amplitude $\mathrm{E}_{0}$ and frequency $\omega$.

A general property of confined electromagnetic waves is the proportionality between energy and frequency. As an example we choose the confinement of light between two perfect reflecting mirrors with surface A. When we press both mirrors together of a distance $\Delta \mathrm{x}$, the work $\Delta \mathrm{W}$ has been delivered:

$$
\Delta \mathrm{W}=\mathrm{p} \mathrm{A} \Delta \mathrm{x}
$$

In which " $A$ " equals the surface of the mirrors and " $p$ " equals the radiation pressure:

$$
\mathrm{p}=\mathrm{w}=\frac{1}{2} \varepsilon \mathrm{E}^{2}+\frac{1}{2} \mu \mathrm{H}^{2}\left[\mathrm{~N} / \mathrm{m}^{2}\right]
$$

The Electromagnetic "Work $\Delta \mathrm{W}$ " which has been delivered equals:

$$
\Delta \mathrm{W}=\mathrm{W} A \Delta \mathrm{x}
$$

When over a time " $\Delta \mathrm{t}$ " both mirrors move towards each other with a velocity " $v$ ", then the distance " $\Delta \mathrm{x}$ " passed over a time interval $\Delta \mathrm{t}$ equals:

$$
\Delta \mathrm{x}=\mathrm{v} \Delta \mathrm{t} \quad[\mathrm{m}]
$$

Substituting (62) in (61) results in:

$$
\begin{aligned}
& \Delta \mathrm{W}=\mathrm{w} \mathrm{A} \Delta \mathrm{x} \\
& \Delta \mathrm{W}=\mathrm{wA} \mathrm{v} \Delta \mathrm{t} \\
& \mathrm{v}=\frac{\Delta \mathrm{W}}{\mathrm{wA} \Delta \mathrm{t}} \\
& 38
\end{aligned}
$$


Due to the Doppler-Shift, the frequency of the confined radiation will increase when the mirrors move towards each other and will decrease when both mirrors move apart from each other. When both mirrors move towards each other, the observed frequency shift $\Delta \mathrm{f}$ will be:

$\Delta \mathrm{f}=\mathrm{f}_{1}-\mathrm{f}_{0}=\mathrm{f}_{0} \sqrt{\frac{1+\frac{v}{c}}{1-\frac{v}{c}}}-\mathrm{f}_{0}=\mathrm{f}_{0}\left(\sqrt{\frac{1+\frac{v}{c}}{1-\frac{v}{c}}}-1\right)$

Applying Taylor series, the observed frequency shift equals:

$$
\begin{aligned}
& \Delta \mathrm{f}=\mathrm{f}_{0}\left(\sqrt{\frac{1+\frac{v}{c}}{1-\frac{v}{c}}}-1\right)=\mathrm{f}_{0}\left(1+\frac{v}{c}+\frac{1}{2}\left(\frac{v}{c}\right)^{2}+\frac{1}{2}\left(\frac{v}{c}\right)^{3}+\frac{3}{8}\left(\frac{v}{c}\right)^{4}+\right)+ \\
& +\mathrm{f}_{0}\left(\frac{3}{8}\left(\frac{v}{c}\right)^{5}+\frac{5}{16}\left(\frac{v}{c}\right)^{6}+\frac{5}{16}\left(\frac{v}{c}\right)^{7}+\frac{35}{128}\left(\frac{v}{c}\right)^{8}+\frac{35}{128}\left(\frac{v}{c}\right)^{9}+\frac{63}{256}\left(\frac{v}{c}\right)^{10}+\ldots .\right)
\end{aligned}
$$

From 


$$
\begin{aligned}
& \Delta \mathrm{f}=\mathrm{f}_{0}\left(1+\frac{v}{c}+\frac{1}{2}\left(\frac{v}{c}\right)^{2}+\frac{1}{2}\left(\frac{v}{c}\right)^{3}+\frac{3}{8}\left(\frac{v}{c}\right)^{4}-1\right) \\
& \Delta \mathrm{f}=\mathrm{f}_{0}\left(\frac{v}{c}+\frac{1}{2}\left(\frac{v}{c}\right)^{2}+\frac{1}{2}\left(\frac{v}{c}\right)^{3}+\frac{3}{8}\left(\frac{v}{c}\right)^{4}\right)
\end{aligned}
$$

At low velocities $(\mathrm{v} \ll \mathrm{c})$

$\mathrm{v} \approx c \frac{\Delta \mathrm{f}}{\mathrm{f}_{0}} \approx \frac{\Delta \mathrm{W}}{\mathrm{WA} \Delta \mathrm{t}}$

$\Delta \mathrm{f} \approx\left(\frac{\mathrm{f}_{0}}{\mathrm{WAc} \Delta \mathrm{t}}\right) \Delta \mathrm{W}=\left(\frac{\mathrm{f}_{0}}{\mathrm{~W}}\right) \Delta \mathrm{W}$

$\Delta \mathrm{W}=\mathrm{h}_{\mathrm{EM}} \Delta \mathrm{f}$

In which "W" equals the total energy involved in the transformation of Mechanical Energy into the Electromagnetic Energy. The term $\mathrm{h}_{\mathrm{EM}}$ represents an Electromagnetic equivalent for Planck's constant.

$$
\begin{aligned}
& \Delta \mathrm{W}=\mathrm{h}_{\mathrm{EM}} \Delta \mathrm{f} \\
& \mathrm{h}_{\mathrm{EM}}=\frac{\mathrm{f}_{0}}{\mathrm{~W}}
\end{aligned}
$$




\section{The Fundamental Discussion between Newton and Einstein}

Einstein's two fundamental contributions to Modern Physics are General Relativity and the theory describing "Gravitational Redshift" based on General Relativity.

Einstein assumes a deformation of the space-time continuum because of a gravitational field. But in general a deformation of any medium will be caused by the change of the energy density within the medium. Like the speed of sound will increase/ decrease when we change the air pressure. However, the speed of sound (which became higher or lower) will still be the same in any direction. The change of the speed of sound will be omni-directional.

A gravitational field contains a gravitational energy-density. For that reason the change in the speed of light will be omnidirectional within a gravitational field (with a omnidirectional gravitational energy density). Einstein however assumes a one-directional change in the speed of light, (only in the direction of the gravitational field). When the change of the speed of light was omni-directional, a beam of light would never be deflected by a gravitational field which is in contradiction with what we measure. Only the absolute value of the speed of light would change omni-directional.

The theory of Newton however results in the theory of a 2directional inertia of photons. The inertia of photons equals zero only in the direction of propagation. Perpendicular to the direction of propagation the mass density of photons is according Einstein's $E=m c^{\wedge} 2$ ).

The inertia of photons [Ref. 7] in the direction of propagation will not change within a gravitational field. Gravity can only interact with mass (inertia). Because the 41 
mass of the photons in the direction of propagation equals zero, there will ne no interaction with the gravitational field and the photon in the direction of propagation. The speed of light in the direction of propagation will remain unaltered. But according Newton, the photon will have inertia (mass) in the directions perpendicular to the direction of propagation and for that reason the photon will interact with the gravitational field and the photon will be deflected, only in the direction of the gravitational field.

And that leads to the consequence that photons will be deflected within a gravitational field when the direction of the gravitational field is perpendicular to the direction of propagation of the photons.

To find fundamental mathematical evidence for this concept, we have to make use of Quantum Light Theory. Quantum Light Theory (QLT) is the development in Quantum Field Theory (QFT). In Quantum Field Theory, the fundamental interaction fields are replacing the concept of elementary particles in Classical Quantum Mechanics. In Quantum Light Theory the fundamental interaction fields are being replaced by One Single Field. The Electromagnetic Field, generally well known as Light. To realize this theoretical concept, the fundamental theory has to go back in time 300 years, the time of Isaac Newton to follow a different path in development.

Nowadays experiments question more and more the fundamental concepts in Quantum Field Theory and Classical Quantum Mechanics. The publication "Operational Resource Theory of Imaginarity" in "Physical Review Letters" in 2021 (Ref. [2]) presenting the first experimental evidence for the measurability of "Quantum Mechanical Imaginarity" directly leads to the fundamental question in this experiment: How is it possible to measure the imaginary part of "Quantum 
Physical Probability Waves"? This publication provides an unambiguously answer to this fundamental question in Physics, based on the fundamental "Gravitational Electromagnetic Interaction" force densities. The "Quantum Light Theory" presents a new "GravitationalElectromagnetic Equation" describing Electromagnetic Field Configurations which are simultaneously the Mathematical Solutions for the Quantum Mechanical "Schrodinger Wave Equation" and more exactly the Mathematical Solutions for the "Relativistic Quantum Mechanical Dirac Equation". The Mathematical Solutions for the "GravitationalElectromagnetic Equation" carry Mass, Electric Charge and Magnetic Spin at discrete values.

\subsection{Newton's approach to General Relativity based on Newtonian Physics.}

To define the Fundamental Equation for the Interaction between Gravity and Light, an extra term (B-6) has been introduced in equation (24). The term B-6 represents the force density of the gravitational field acting on the electromagnetic mass density.

$$
\mathrm{F}_{\text {GRAVITY }}=\mathrm{m} \overline{\mathrm{g}}[\mathrm{N}]
$$

Dividing both terms by the Volume V:

$$
\frac{\mathrm{F}_{\text {GRAVITY }}}{\mathrm{V}}=\frac{\mathrm{m}}{\mathrm{V}} \overline{\mathrm{g}}\left[\mathrm{N} / \mathrm{m}^{3}\right]
$$

Results in the force density:

$$
\mathrm{f}_{\text {GRAVITY }}=\rho \overline{\mathrm{g}} \quad\left[\mathrm{N} / \mathrm{m}^{3}\right]
$$

The specific mass " $\rho$ " of a beam of light follows from Einstein's equation: 
$\mathrm{W}=\mathrm{m} \mathrm{c}^{2}$

Divinding both terms by the Volume V results in:

$\frac{\mathrm{W}}{\mathrm{V}}=\frac{\mathrm{m}}{\mathrm{V}} \mathrm{c}^{2}$

which represents the energy density "w" and the specific

mass " $\rho$ " of the electromagnetic radiation:

$\mathrm{w}=\rho \mathrm{c}^{2}$

which results for an expression of the specific mass $\rho$ :

$\rho=\frac{1}{\mathrm{c}^{2}} \mathrm{~W}=\varepsilon \mu \mathrm{w}$

The energy density " $w$ " follows from the electric and the magnetic field intensities:

$$
\begin{aligned}
& \mathrm{w}=\frac{1}{2} \varepsilon \mathrm{E}^{2}+\frac{1}{2} \mu \mathrm{H}^{2} \\
& \mathrm{w}=\frac{1}{2}\left(\varepsilon \mathrm{E}^{2}+\mu \mathrm{H}^{2}\right)=\frac{1}{2}(\varepsilon(\overline{\mathrm{E}} \cdot \overline{\mathrm{E}})+\mu(\overline{\mathrm{H}} \cdot \overline{\mathrm{H}}))
\end{aligned}
$$

Substituting equation (69) and (70) in equation (68) results in the gravitational force density f fRAVITY acting on an arbitrary electromagnetic field configuration (a beam of light) with mass density $\rho$.

$$
\begin{aligned}
& \mathrm{f}_{\text {GRAVITY }}=\rho \overline{\mathrm{g}} \\
& \mathrm{f}_{\text {GRAVITY }}=\rho \overline{\mathrm{g}}=\varepsilon \mu \mathrm{w} \overline{\mathrm{g}}=\frac{1}{2}\left(\varepsilon^{2} \mu(\overline{\mathrm{E}} \cdot \overline{\mathrm{E}})+\varepsilon \mu^{2}(\overline{\mathrm{H}} \cdot \overline{\mathrm{H}})\right) \overline{\mathrm{g}}
\end{aligned}
$$

Substituting equation (71) in equation (47) results in the fundamental equation describing the ElectromagneticGravitational interaction for any arbitrary electromagnetic field configuration (a beam of light): 


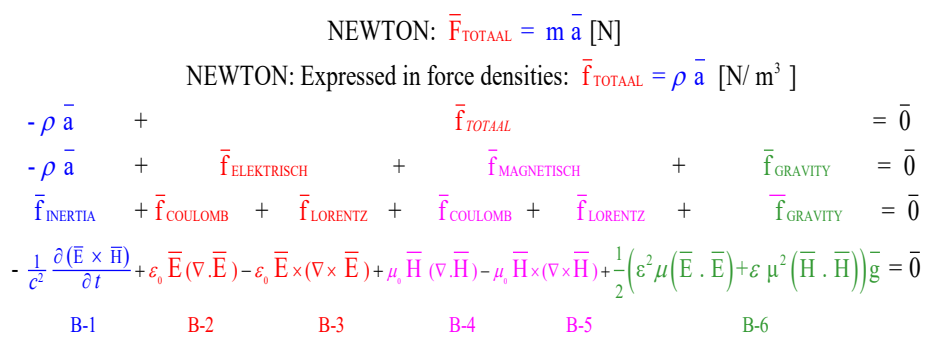

Term B-1 represents the inertia term of the electromagnetic radiation. Term B-4 is the magnetic representation of the (electric) Coulomb's Force B-2 and Term B-3 is the electric representation of the (magnetic) Lorentz Force B-5. Term B6 represents the Electromagnetic-Gravitational interaction of a gravitational field with field acceleration $\bar{g}$ acting on an arbitrary electromagnetic field configuration ( a beam of light) with specific mass $\rho$.

The universal equation for the electromagnetic field (free electromagnetic waves and confined electromagnetic fields) within a gravitational field with gravity field intensity $\bar{g}$ has been presented in (72) and expresses the perfect equilibrium between the inertia forces (B-1), the electric forces (B-2 and B-3), the magnetic forces (B-4 and B-5) and the gravitational force (B-6) in any arbitrary electromagnetic field configuration.

$$
\begin{gathered}
-\frac{1}{c^{2}} \frac{\partial(\overline{\mathrm{E}} \times \overline{\mathrm{H}})}{\partial t}+\varepsilon_{0} \overline{\mathrm{E}}(\nabla \cdot \overline{\mathrm{E}})-\varepsilon_{0} \overline{\mathrm{E}} \times(\nabla \times \overline{\mathrm{E}})+\mu_{0} \overline{\mathrm{H}}(\nabla \cdot \overline{\mathrm{H}})- \\
\text { B-1 } \quad \text { B-2 } \quad \text { B-3 } \\
-\mu_{0} \overline{\mathrm{H}} \times(\nabla \times \overline{\mathrm{H}})+\frac{1}{2}\left(\varepsilon^{2} \mu(\overline{\mathrm{E}} \cdot \overline{\mathrm{E}})+\varepsilon \mu^{2}(\overline{\mathrm{H}} \cdot \overline{\mathrm{H}})\right) \overline{\mathrm{g}}=\overline{0}
\end{gathered}
$$

\section{B-6}


The "event horizon" is the boundary defining the region of space around a black hole from which nothing (not even light) can escape. The "event horizon" has been created by the equilibrium between the gravitational forces and the forces of electromagnetic interaction and the radiation pressure. At the boundary of a Black Hole does exist the prefect equilibrium. There are two types of Black Holes.

The first type represents an equilibrium in which the Black Hole has been represented by a hollow sphere. Only within the extreme thin shell of the hollow sphere does exist a perfect equilibrium. Inside the hollow sphere any kind of light (electromagnetic radiation) has been pressed with an extreme high radiation pressure towards to the "Shell of Perfect Equilibrium" (Event Horizon) because the radiation pressure inside the hollow sphere is always larger than the confining gravitational force. Outside the hollow sphere, any kind of light (electromagnetic radiation) has been attracted towards the "Shell of Perfect Equilibrium" (Event Horizon) because outside the hollow sphere the confining gravitational force is always larger than the radiation pressure. This type has been called "Transversal Black Holes". Because like a planet moves within an orbit around a star, any beam of light (electromagnetic radiation) propagates within the Shell of Perfect Equilibrium" (Event Horizon) in an orbit around the Black Hole.

The second type represents an Equilibrium everywhere around the black Hole. At a special distance from the centre of the Black Hole, the radiation density increases suddenly very sharp. This has been called the Boundary (Event Horizon) around the Black Hole. This type has been called "Longitudinal Black Holes". Because the light (electromagnetic radiation) moves towards the Black Holes or away from the Black Hole in the same direction as the 
direction of the Gravitational Field generated by the Black Hole.

We consider a beam of light passing a strong gravitational field, generated by a Black Hole. According the first term in (1) the beam of light will follow a circular orbit around the Black Hole. The required Equilibrium will exist at the radius where the centrifugal electromagnetic inertia forces will be equal and opposite directed to the centripetal oriented gravitational forces on the electromagnetic mass. Figure 12 represents the orbit (colored red) of a LASER beam around a uniform intense gravitational field (Black Hole) .

In general, Newtons second law of motion has been presented as:

$\mathrm{F}=\mathrm{ma}$

In which "a" represents the acceleration which equals the difference of the velocity $\Delta \mathrm{v}$ divided by the time interval $\Delta \mathrm{t}$.

$$
\mathrm{a}=\frac{\Delta \mathrm{v}}{\Delta \mathrm{t}}
$$

The momentum $p$ of a mechanical mass equals:

$$
\mathrm{p}=\mathrm{m} \mathrm{v}
$$

Then Newton's second law of motion can be presented as:

$$
\mathrm{F}_{\text {INERTIA }}=\mathrm{m} \mathrm{a}=\mathrm{m} \frac{\Delta \mathrm{v}}{\Delta \mathrm{t}}=\frac{\Delta(\mathrm{m} \mathrm{v})}{\Delta \mathrm{t}}=\frac{\Delta(\mathrm{p})}{\Delta \mathrm{t}}
$$


Like a mechanical mass expresses the property of inertia, also a beam of light expressed the property of inertia. When the sun shines on the earth, the radiation of the sun presses on the earth with thousands of Newton.

Like a mechanical mass, also a beam of light has momentum. The momentum of a beam of light has been expressed by the Poynting vector $\mathrm{S}$ and equals the mechanical momentum vector $\mathrm{p}$ multiplied by the square of the speed of light $\mathrm{c}$ divided by the Volume.

$$
\begin{gathered}
\text { (mechanical mass) } \\
\mathrm{F}_{\text {INERTIA }}=\mathrm{m} \mathrm{a}=\mathrm{m} \frac{\Delta \mathrm{v}}{\Delta \mathrm{t}}=\frac{\Delta(\mathrm{m} \mathrm{v})}{\Delta \mathrm{t}}=\frac{\Delta(\mathrm{p})}{\Delta \mathrm{t}}=\frac{\mathrm{V}}{\mathrm{c}^{2}} \frac{\Delta(\mathrm{S})}{\Delta \mathrm{t}}
\end{gathered}
$$

The inertia force density "f" equals the inertia force "F" divided by the Volume "V".

(mechanical mass) (beam of light)

$$
f_{\text {INERTIA }}=\left(\frac{\mathrm{m}}{\mathrm{V}}\right) \mathrm{a}=\rho \frac{\Delta \mathrm{v}}{\Delta \mathrm{t}}=\frac{1}{\mathrm{c}^{2}} \frac{\Delta(\mathrm{S})}{\Delta \mathrm{t}}
$$

The well-known equation of Einstein equals:

$$
\begin{aligned}
& \mathrm{W}=\mathrm{mc}^{2} \\
& \mathrm{w}=\frac{\mathrm{W}}{\mathrm{V}}=\frac{\mathrm{m}}{\mathrm{V}} \mathrm{c}^{2}=\rho \mathrm{c}^{2}
\end{aligned}
$$

In which "w" represents the electromagnetic energy density and equals:

$$
\mathrm{w}=\frac{1}{2} \varepsilon \mathrm{E}^{2}+\frac{1}{2} \mu \mathrm{H}^{2}
$$

For electromagnetic radiation the electromagnetic impedance $Z_{0}$ equals: 


$$
\begin{aligned}
& \mathrm{Z}_{0}=\frac{\mathrm{E}}{\mathrm{H}}=\sqrt{\frac{\mu}{\varepsilon}} \\
& \mathrm{H}=\mathrm{E} \sqrt{\frac{\varepsilon}{\mu}} \\
& \mathrm{w}=\frac{1}{2} \varepsilon \mathrm{E}^{2}+\frac{1}{2} \mu \mathrm{H}^{2}=\frac{1}{2} \varepsilon \mathrm{E}^{2}+\frac{1}{2} \varepsilon \mathrm{E}^{2}=\varepsilon \mathrm{E}^{2}
\end{aligned}
$$

Substituting equation (66) in (64) results in:

$$
\begin{aligned}
& \mathrm{w}=\rho \mathrm{c}^{2}=\varepsilon \mathrm{E}^{2} \\
& \rho=\frac{\varepsilon}{\mathrm{c}^{2}} \mathrm{E}^{2}
\end{aligned}
$$

Because the beam of light has been confined in the radial direction, it demonstrates in the radial direction the property of inertia (electromagnetic mass) and interacts with a gravitational field according Newton's second law of motion. The whole Universe is in a perfect Equilibrium. Also at the "Event Horizon" of a Black Hole does exist a perfect equilibrium between the confining gravitational force of the Black Hole and the radial directed inertia force density of the confined electromagnetic radiation (Laser Beam confined by a Black Hole at the "Event Horizon"). To determine the "Event Horizon" of the Black Hole (Radius of the circular orbit of the Laser Beam), we have to find the perfect equilibrium between the inertia force densities of the electromagnetic energy densities of the Laser Beam and the confining gravitational force acting on the electromagnetic energy densities of the Laser Beam.

$$
\begin{aligned}
& \mathrm{f}_{\text {GRAVITY }}=\mathrm{f}_{\text {INERTIA }} \\
& \rho \mathrm{g}=\frac{1}{c^{2}} \frac{\Delta(\mathrm{S})}{\Delta \mathrm{t}}
\end{aligned}
$$




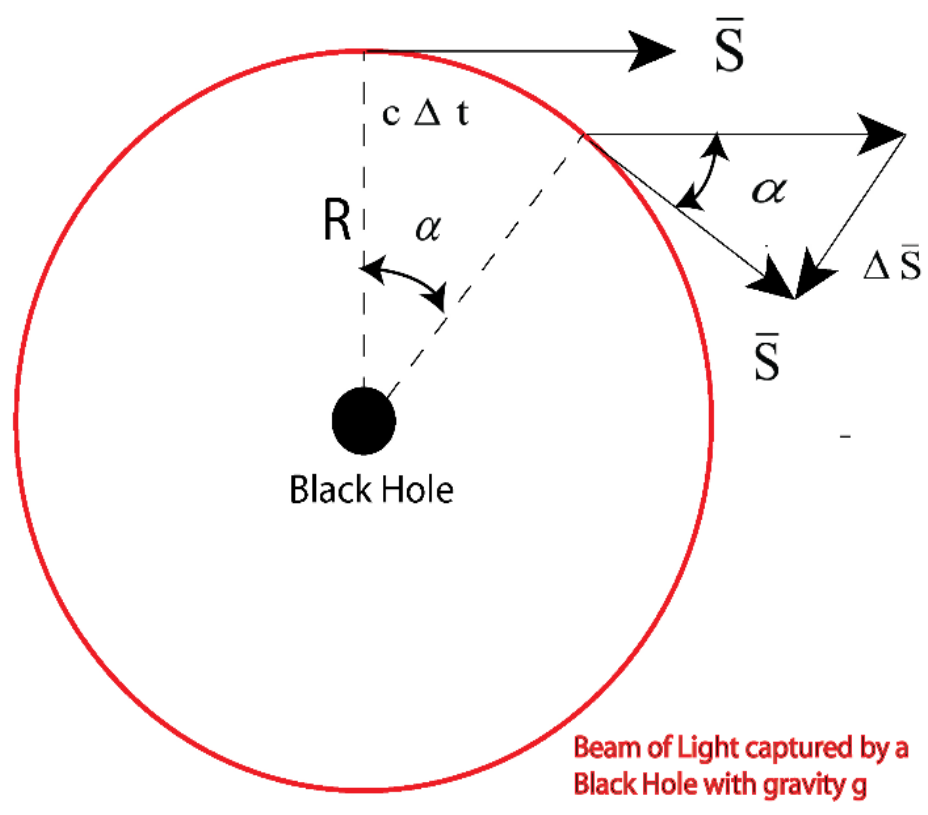

Fig. 6 A LASER beam around a Black Hole captured in a circular orbit around the Black Hole in a Transversal Modus by the Gravitational interaction of the Black Hole with the mass (inertia) of the Laser Beam Light.

From Figure (5) follows the relationship between de changing in the Poynting vector $\Delta \mathrm{S}$ and the time interval $\Delta \mathrm{t}$ :

$\operatorname{Tan}(\alpha)=\frac{\mathrm{c} \Delta \mathrm{t}}{\mathrm{R}}=\frac{\Delta \mathrm{S}}{\mathrm{S}}$

$\frac{\Delta \mathrm{S}}{\Delta \mathrm{t}}=\frac{\mathrm{c} \mathrm{S}}{\mathrm{R}}$

$\rho g=\frac{1}{c^{2}} \frac{\Delta(S)}{\Delta t}=\frac{1}{c^{2}} \frac{c S}{R}=\frac{1}{c} \frac{S}{R}$ 
The Poynting vector $\overline{\mathrm{S}}$ represents the total energy transport of the electromagnetic radiation per unit surface per unit time $[\mathrm{J}$ $\left./ \mathrm{m}^{2} \mathrm{~s}\right]$. Which can be written as the cross product of the Electric Field intensity $\bar{E}$ and the magnetic Field intensity $\bar{H}$.

$$
\begin{aligned}
& \overline{\mathrm{S}}=\overline{\mathrm{E}} \times \overline{\mathrm{H}} \\
& \mathrm{S}=\mathrm{E} \mathrm{H} \operatorname{Sin}\left(90^{\circ}\right)=\mathrm{E} H \\
& S=\mathrm{E}^{2} \sqrt{\frac{\varepsilon}{\mu}}
\end{aligned}
$$

Substituting equation (10) and (13) in (12) results in an equation for the Event Horizon at radius "R" of a Transversal Black Hole.

$$
\begin{aligned}
& \rho \mathrm{g}=\frac{1}{\mathrm{c}} \frac{\mathrm{S}}{\mathrm{R}} \\
& \mathrm{R}=\frac{\mathrm{S}}{\rho \mathrm{cg}} \\
& R=\frac{\mathrm{E}^{2} \sqrt{\frac{\varepsilon}{\mu}}}{\rho \mathrm{cg}}=\frac{\mathrm{E}^{2} \sqrt{\frac{\varepsilon}{\mu}}}{\frac{\varepsilon}{\mathrm{c}} \mathrm{E}^{2} \mathrm{~g}} \\
& \mathrm{R}=\frac{\mathrm{c}^{2}}{\mathrm{~g}} \approx \frac{910^{16}}{g}[\mathrm{~m}]
\end{aligned}
$$

Equation (84) represents the perfect equilibrium between the inertia force densities of the electromagnetic mass $\frac{1}{c^{2}} \frac{\Delta \overline{\mathrm{S}}}{\Delta \mathrm{t}}$ and the centripetal oriented gravitational force density $\frac{\mathrm{w}}{\mathrm{c}^{2}} \mathrm{~g}$ acting on the electromagnetic mass. The perfect equilibrium direction where the inertia forces due to the circular orbit of the beam of light are in a perfect balance with the attractive gravitational 
forces, exists at one defined radius " $R$ " of the beam of light (LASER beam), independent of the intensity of the beam of light and independent of the frequency of the beam of light. Only the acceleration " $g$ " of the gravitational field determines the radius of equilibrium " $R$ "

$$
\mathrm{R} \approx \frac{910^{16}}{g}
$$

In which " $R$ " is the radius of the beam of light and " $g$ " the acceleration of the gravitational field of the "Black Hole".

The $\mathrm{x}-\mathrm{y}$ plane is oriented perpendicular on the z-direction. The speed of light towards the positive $z$-direction equals the speed of light (the constant "c $=300.000 \mathrm{~km} / \mathrm{s}$ "). But the speed of light in the $x-y$ plane has to be exactly zero $[9,14,15]$. Else the diameter of the laser beam would become larger and larger during the propagation along the positive z-direction. This is only possible because the Electromagnetic confining forces B2, B-3, B-4 and B-5 in Equation (28) compensate exactly the outward oriented radiation pressure towards the $\mathrm{x}$-direction and the y-direction.

The Electric Radiation Pressure has been compensated completely by the Coulomb Force Densities within the Laser Beam. At the same time the inertia force density within the beam of light has been fully compensation by the gravitational force density (generated by the black hole) comparable with a satellite propagating around the earth.

The Newtonian approach results in a Radius "R" (72) around a Black Hole comparable with the results in General Relativity. It is not necessary to develop a new theory in 
which space and time are part of a curved space-time continuum. 


\subsection{Newton's approach to "Gravitational Redshift" based on Newtonian Physics.}

To describe gravitational Redshift we have to start with Planck's Law describing the proportionality between Energy and Frequency.

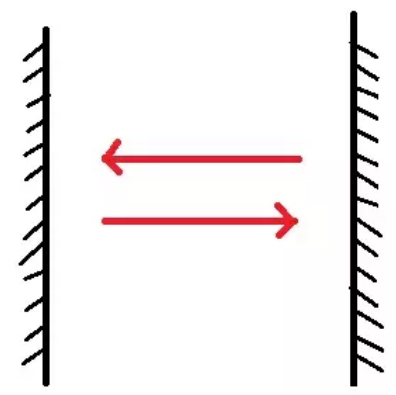

Figure 7 Electromagnetic radiation confined between two $100 \%$ reflecting mirrors

Figure 7 represents confined electromagnetic radiation with amplitude $\mathrm{E}_{0}$ and frequency $\omega$.

A general property of confined electromagnetic waves is the proportionality between energy and frequency. As an example we choose the confinement of light between two perfect reflecting mirrors with surface $A$. When we press both mirrors together of a distance $\Delta \mathrm{x}$, the work $\Delta \mathrm{W}$ has been delivered:

$\Delta \mathrm{W}=\mathrm{p} \mathrm{A} \Delta \mathrm{x}$

In which " $A$ " equals the surface of the mirrors and " $p$ " equals the radiation pressure:

$$
\mathrm{p}=\mathrm{w}=\frac{1}{2} \varepsilon \mathrm{E}^{2}+\frac{1}{2} \mu \mathrm{H}^{2}\left[\mathrm{~N} / \mathrm{m}^{2}\right]
$$


The Electromagnetic "Work $\Delta \mathrm{W}$ " which has been delivered equals:

$$
\Delta \mathrm{W}=\mathrm{wA} \Delta \mathrm{x}
$$

When over a time " $\Delta \mathrm{t}$ " both mirrors move towards each other with a velocity " $\mathrm{v}$ ", then the distance " $\Delta \mathrm{x}$ " passed over a time interval $\Delta \mathrm{t}$ equals:

$$
\Delta \mathrm{x}=\mathrm{v} \Delta \mathrm{t} \quad[\mathrm{m}]
$$

Substituting (92) in (93) results in:

$$
\begin{aligned}
& \Delta \mathrm{W}=\mathrm{wA} \Delta \mathrm{x} \\
& \Delta \mathrm{W}=\mathrm{wA} \Delta \mathrm{t} \\
& \mathrm{v}=\frac{\Delta \mathrm{W}}{\mathrm{wA} \Delta \mathrm{t}}
\end{aligned}
$$

Due to the Doppler-Shift, the frequency of the confined radiation will increase when the mirrors move towards each other and will decrease when both mirrors move apart from each other. When both mirrors move towards each other, the observed frequency shift $\Delta \mathrm{f}$ will be:

$$
\Delta \mathrm{f}=\mathrm{f}_{1}-\mathrm{f}_{0}=\mathrm{f}_{0} \sqrt{\frac{1+\frac{v}{c}}{1-\frac{v}{c}}}-\mathrm{f}_{0}=\mathrm{f}_{0}\left(\sqrt{\frac{1+\frac{v}{c}}{1-\frac{v}{c}}}-1\right)
$$

Applying Taylor series, the observed frequency shift equals: 


$$
\begin{aligned}
& \Delta \mathrm{f}=\mathrm{f}_{0}\left(\sqrt{\frac{1+\frac{v}{c}}{1-\frac{v}{c}}}-1\right)=\mathrm{f}_{0}\left(-1+1+\frac{v}{c}+\frac{1}{2}\left(\frac{v}{c}\right)^{2}+\frac{1}{2}\left(\frac{v}{c}\right)^{3}+\frac{3}{8}\left(\frac{v}{c}\right)^{4}+\right)+ \\
& +\mathrm{f}_{0}\left(\frac{3}{8}\left(\frac{v}{c}\right)^{5}+\frac{5}{16}\left(\frac{v}{c}\right)^{6}+\frac{5}{16}\left(\frac{v}{c}\right)^{7}+\frac{35}{128}\left(\frac{v}{c}\right)^{8}+\frac{35}{128}\left(\frac{v}{c}\right)^{9}+\frac{63}{256}\left(\frac{v}{c}\right)^{10}+\ldots .\right)
\end{aligned}
$$

\section{$\underline{\underline{\text { rom }}}$}

$$
\begin{aligned}
& \Delta \mathrm{f}=\mathrm{f}_{0}\left(1+\frac{v}{c}+\frac{1}{2}\left(\frac{v}{c}\right)^{2}+\frac{1}{2}\left(\frac{v}{c}\right)^{3}+\frac{3}{8}\left(\frac{v}{c}\right)^{4}-1\right) \\
& \Delta \mathrm{f}=\mathrm{f}_{0}\left(\frac{v}{c}+\frac{1}{2}\left(\frac{v}{c}\right)^{2}+\frac{1}{2}\left(\frac{v}{c}\right)^{3}+\frac{3}{8}\left(\frac{v}{c}\right)^{4}\right)
\end{aligned}
$$

At low velocities $(\mathrm{v} \ll \mathrm{c})$

$$
\mathrm{v} \approx c \frac{\Delta \mathrm{f}}{\mathrm{f}_{0}} \approx \frac{\Delta \mathrm{W}}{\mathrm{w} \mathrm{A} \Delta \mathrm{t}}
$$

$\Delta \mathrm{f} \approx\left(\frac{\mathrm{f}_{0}}{\mathrm{WAc} \Delta \mathrm{t}}\right) \Delta \mathrm{W}=\left(\frac{\mathrm{f}_{0}}{\mathrm{~W}}\right) \Delta \mathrm{W}$

$\Delta \mathrm{W}=\mathrm{h}_{\mathrm{EM}} \Delta \mathrm{f}$

In which "W" equals the total energy involved in the transformation of Mechanical Energy into the Electromagnetic Energy. The term $\mathrm{h}_{\mathrm{EM}}$ represents an Electromagnetic equivalent for Planck's constant.

$$
\begin{aligned}
& \Delta \mathrm{W}=\mathrm{h}_{\mathrm{EM}} \Delta \mathrm{f} \\
& \mathrm{h}_{\mathrm{EM}}=\frac{\mathrm{f}_{0}}{\mathrm{~W}}
\end{aligned}
$$

The "Newtonian Equilibrium Equation for Electromagnetic Waves interacting with a Gravitational Field with acceleration $\bar{g}$ ", 
has been presented in Chapter1: "The Fundamental Discussion between Newton and Maxwell": in Equation (47):

$$
\begin{gathered}
\text { 3-Dimensional Space Domain } \\
\left(\begin{array}{c}
\mathrm{x}_{3}-1 \\
\mathrm{x}_{2} \\
\mathrm{x}_{1}
\end{array}\right)+\mu_{0} \overline{\mathrm{H}}(\nabla \cdot \overline{\mathrm{H}})-\mu_{0} \overline{\mathrm{H}} \times(\nabla \times \overline{\mathrm{H}})+\frac{1}{2}\left(\varepsilon^{2} \mu(\overline{\mathrm{E}} \cdot \overline{\mathrm{E}})+\varepsilon \mu^{2}(\overline{\mathrm{H}} \cdot \overline{\mathrm{H}})\right) \overline{\mathrm{g}}=\overline{0} \\
c^{\mathrm{B}-4} \frac{\partial(\overline{\mathrm{E}} \times \overline{\mathrm{H}})}{\partial t}+\varepsilon_{0} \overline{\mathrm{E}}(\nabla \cdot \overline{\mathrm{E}})-\varepsilon_{0} \overline{\mathrm{E}} \times(\nabla \times \overline{\mathrm{E}})+
\end{gathered}
$$

A fundamental Solution for Equation (99) within a gravitational constant gravitational field $\mathrm{g}$ in the $\mathrm{z}$-direction, equals for the electric field:

$$
\overline{\mathrm{E}}=\left(\begin{array}{c}
\mathrm{e}_{x} \\
\mathrm{e}_{y} \\
\mathrm{e}_{z}
\end{array}\right)=\left(\begin{array}{c}
\mathrm{e}^{-\frac{1}{2} \mathrm{gz} \mu_{0} \varepsilon_{0}} \mathrm{~h}[\mathrm{t}-\mathrm{z} \sqrt{\varepsilon \mu}] \\
0 \\
0
\end{array}\right)=\left(\begin{array}{c}
\mathrm{e}^{-\frac{1}{2} \mathrm{gz} \mu_{0} \varepsilon_{0}} \mathrm{~h}\left[\mathrm{t}-\frac{\mathrm{k}_{z}}{\omega} \mathrm{z}\right] \\
0 \\
0
\end{array}\right)
$$

And for the Magnetic Field:

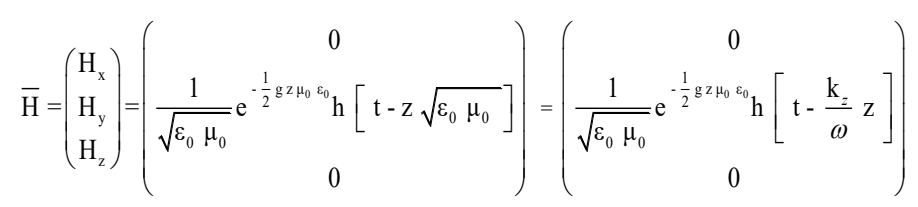

According Planck's law for electromagnetic waves, the Newtonian presentation for the Gravitational Frequency Shift (Red-Shift) equals: 
$\overline{\mathrm{E}}=\left(\begin{array}{l}\mathrm{e}_{x} \\ \mathrm{e}_{y} \\ \mathrm{e}_{z}\end{array}\right)=\left(\begin{array}{c}\mathrm{e}^{-\frac{1}{2} g z \mu_{0} \varepsilon_{0}} \mathrm{~h}\left[\mathrm{t}-\frac{\mathrm{k}_{z} \mathrm{e}^{-g z \mu_{0} \varepsilon_{0}}}{\omega \mathrm{e}^{-\mathrm{g} z \mu_{0} \varepsilon_{0}}} \mathrm{z}\right. \\ 0 \\ 0\end{array}\right)$

And for the Magnetic Field:

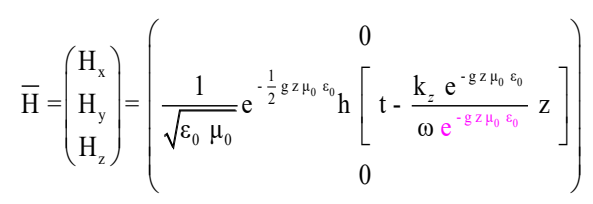

Equations (102) and (103) represent a "Constant Speed of Light" "c" In which the "Newtonian Gravitational Red-Shift" equals:

$\omega_{\text {Newtonian Gravitational Redshift }}=\omega_{0} \mathrm{e}^{-\mathrm{gz} \mu_{0} \varepsilon_{0}}$

In which $\omega_{0}$ equals the original frequency of the beam of light parallel to the constant gravitational field in the $\mathrm{z}$ direction and the exponential term demonstrates the Gravitational Redshift when the beam of light moves away from the Gravity Source (Solar System or Black Hole) and represents the Blue-Shift when the beam of light moves towards the Gravity Source. Including the "Relativistic Longitudinal Doppler Effect" results in:

$\omega_{\text {Newtonian Longitidinal Gravitational Redshitt }}=\omega_{0} \mathrm{e}^{-g z \mu_{0} \varepsilon_{0}} \sqrt{\frac{1+\frac{v}{c}}{1-\frac{v}{c}}}$ 
Equation (104) represents the "Longitudinal Gravitational Redshift" for a Constant Gravitational Field. The "Newton Electromagnetic Equilibrium Equation" (99) represents possible solutions for all "Time- and Space Depending Gravitational Fields" and Equation (99) is the fundamental equation to be "tested" relative to Einstein's theory of General Relativity. New measurements of "Longitudinal Gravitational Frequency Shift (Red-Shift)" from World Wide Observatories could falsify or confirm this new theory.

\subsection{Deviations in the "Hubble Constant" Value}

Due to the Doppler-Shift, the observed frequency of the radiation will increase when two objects move towards each other and will decrease when both objects move apart from each other. When both objects move apart from each other, the observed frequency shift $\Delta \mathrm{f}$ will be:

$$
\Delta \mathrm{f}=\mathrm{f}_{1}-\mathrm{f}_{0}=-\mathrm{f}_{0}\left(\sqrt{\frac{1+\frac{v}{c}}{1-\frac{v}{c}}}\right)
$$

Applying Taylor series, the observed frequency shift equals:

$$
\begin{aligned}
& \Delta \mathrm{f}=\mathrm{f}_{0}\left(\sqrt{\frac{1+\frac{v}{c}}{1-\frac{v}{c}}}-1\right)=\mathrm{f}_{0}\left(-1+1+\frac{v}{c}+\frac{1}{2}\left(\frac{v}{c}\right)^{2}+\frac{1}{2}\left(\frac{v}{c}\right)^{3}+\frac{3}{8}\left(\frac{v}{c}\right)^{4}+\right)+ \\
& +\mathrm{f}_{0}\left(\frac{3}{8}\left(\frac{v}{c}\right)^{5}+\frac{5}{16}\left(\frac{v}{c}\right)^{6}+\frac{5}{16}\left(\frac{v}{c}\right)^{7}+\frac{35}{128}\left(\frac{v}{c}\right)^{8}+\frac{35}{128}\left(\frac{v}{c}\right)^{9}+\frac{63}{256}\left(\frac{v}{c}\right)^{10}+\ldots .\right)
\end{aligned}
$$


Which approximates:

$$
\begin{aligned}
& \Delta \mathrm{f}_{\text {Doppler }}=-\mathrm{f}_{0}\left(1+\frac{v}{c}+\frac{1}{2}\left(\frac{v}{c}\right)^{2}+\frac{1}{2}\left(\frac{v}{c}\right)^{3}+\frac{3}{8}\left(\frac{v}{c}\right)^{4}-1\right) \\
& \Delta \mathrm{f}_{\text {Doppler }}=\mathrm{f}_{0}\left(\frac{v}{c}+\frac{1}{2}\left(\frac{v}{c}\right)^{2}+\frac{1}{2}\left(\frac{v}{c}\right)^{3}+\frac{3}{8}\left(\frac{v}{c}\right)^{4}\right)
\end{aligned}
$$

At low velocities $(\mathrm{v} \ll \mathrm{c})$

$$
\Delta \mathrm{f}_{\text {Doppler }}=\mathrm{f}_{0}\left(\frac{v}{c}+\frac{1}{2}\left(\frac{v}{c}\right)^{2}+\frac{1}{2}\left(\frac{v}{c}\right)^{3}\right)
$$

Taylor series for the Gravitational Red-Shift results in:

$$
\begin{aligned}
\Delta \mathrm{f}_{N G R} & =\omega_{0} \mathrm{e}^{-\mathrm{gz} \mu_{0} \varepsilon_{0}}=\mathrm{f}_{0}\left(-\mathrm{g} \mathrm{z} \mu_{0} \varepsilon_{0}\right)+ \\
& +\frac{1}{2}\left(-\mathrm{g} \mathrm{z} \mu_{0} \varepsilon_{0}\right)^{2}+\frac{1}{6}\left(-\mathrm{g} \mathrm{z} \mu_{0} \varepsilon_{0}\right)^{3}
\end{aligned}
$$

It follows from (109) that the observed Gravitational Frequency Shift is proportional to the distance " $\mathrm{z}$ " between both objects. Under the assumption that the Doppler shift has been caused by a "relative velocity" between both objects, we observe an expanding universe with a "Constant Relative Velocity ( $\left.\mathrm{H}_{0}=67.4 \pm 0.5 \mathrm{~km} \mathrm{~s}^{-1} \mathrm{Mpc}-1\right)$ " in which the "Relative Velocity" between both objects is proportional with the distance between both objects. Under the assumption that the average gravitational field is constant over the distance " $z$ " or gravitational fluctuations counterbalance each other, the conclusion can be drawn that the "Hubble Constant Value" has been measured under the condition of an expanding universe or under the condition of a relative constant gravitational field. Both conditions will result in the same observations (Ref. [11] and [12]). 


$$
\begin{aligned}
& \Delta \mathrm{f}_{N G R}=\omega_{0} \mathrm{e}^{-\mathrm{g} z \mu_{0} \varepsilon_{0}}=\mathrm{f}_{0}\left(-\mathrm{g} \frac{\mathrm{z}}{\mathrm{c}^{2}}\right)+ \\
& +\frac{1}{2}\left(-g \frac{z}{c^{2}}\right)^{2}+\frac{1}{6}\left(-g \frac{z}{c^{2}}\right)^{3} \cdots \cdots \cdots \\
& \Delta \mathrm{f}_{\text {Doppler }}=-\mathrm{f}_{0}\left(\frac{v}{c}+\frac{1}{2}\left(\frac{v}{c}\right)^{2}+\frac{1}{2}\left(\frac{v}{c}\right)^{3}\right) \\
& \Delta \mathrm{H}_{0}=\frac{1}{\mathrm{~V}}\left(\frac{\mathrm{zg}}{\mathrm{c}}\right)\left(\Delta \mathrm{f}_{\text {Doppler }}\right)-\Delta \mathrm{f}_{N G R} \approx \frac{1}{2 \mathrm{c}^{3}} \mathrm{zg} \mathrm{v}-\frac{1}{2 \mathrm{c}^{4}} \mathrm{~g}^{2} \mathrm{z}^{2} \\
& \Delta \mathrm{H}_{0}=\frac{1}{2 \mathrm{c}^{3}} \mathrm{zg}\left(\mathrm{v}-\mathrm{g} \frac{\mathrm{z}}{\mathrm{c}}\right) \approx 210^{-26}\left(\mathrm{v}-\mathrm{g} \frac{\mathrm{z}}{\mathrm{c}}\right)(\mathrm{zg})
\end{aligned}
$$

It follows from (110) that with a measurement accuracy of $10 \%$, for the "Hubble Constant $\mathrm{H}_{0}$ " the first deviation term $\Delta \mathrm{H}_{0}$ in the "Hubble Constant" will appear at distances of about $10^{25}$ [m] (about $1000 \mathrm{Mpc}$ ). This is under the assumption of the absence of strongly disturbing gravitational fields generated by objects like a "Black Hole". In the presence of disturbing objects, higher deviations in the "Hubble Constant" will be measured, which appears in recent measurements by the (HST). In recent measurements for the "Hubble Constant" by the HST, fundamental deviations (Ref. [14] and [15]) in the Hubble Constant has been measured. Being recently observed by the HST, no other theory explains this effect . 


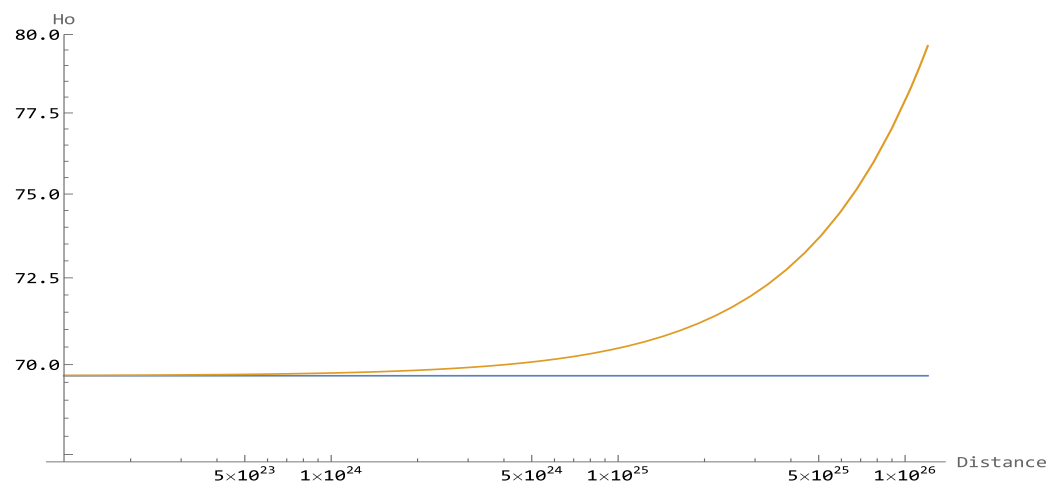

Fig. 8 Plot of measured $\mathrm{H}_{0}$ (Colour Blue) and calculated $\mathrm{H}_{0 \text { (NRS) }}$ (Colour Orange) within a gravitational field of $\mathrm{g}=10^{-10}\left[\mathrm{~m} / \mathrm{s}^{2}\right]$ as function of the Distance " $\mathrm{z}$ " between Object and Observer. 


\section{Conclusions}

It is possible to design GEONs with sizes of sub atomic level $\left(<10^{-10}[\mathrm{~m}]\right)$ and an infinite lifetime which are in perfect equilibrium with themselves and their surrounding. (Equation (51), Figure (4).

Solutions for GEONs (Gravitational Electromagnetic Confinement) always have to be solutions of the relativistic quantum mechanical Dirac Equation (Equation 47.1)

Measuring the imaginary part of the solutions for the relativistic quantum mechanical Dirac Equation is identical with measuring the imaginary part of the quantum mechanical probability waves (de Broglie waves). When the Electromagnetic confined waves for the GEONs have been written in a quantum mechanical complex way:

$$
\bar{\phi}=\frac{1}{\sqrt{2 \mu}}\left(\overline{\mathrm{B}}+\mathrm{i} \frac{\overline{\mathrm{E}}}{\mathrm{c}}\right)
$$

In which " $\mathrm{B}$ " equals the magnetic field component of the electromagnetic GEON field configuration and "E" equals the electric field component of the electromagnetic GEON field configuration). Then is well understandable how the imaginary part of the GEON waves have been measured. Instead of the magnetic part (B), the electric part (E) of the GEONs has been measured.

For this reason the results of the experiments, published in 2021 "Operational Resource Theory of Imaginarity " in "Physical Review Letters"(Ref. [2]) present strong evidence for the existence at sub-atomic level of the electromagnetic GEONs and the correctness of Wheeler's theory. 
The Newtonian approach results in a Radius " $R$ " (88) around a Black Hole comparable with the results in General Relativity. Equation (105) represents the "Longitudinal Gravitational Redshift" for a Constant Gravitational Field including the doppler effect. Accurate observations by the "Hubble Space Telescope" of variations in the "Hubble Constant Value" nearby strong gravitational fields will falsify or confirm the new theory.

The new theory predicts deviations (Ref. [14] - [18]) $\Delta \mathrm{H}_{0}$ (Equation 110) in the "Hubble Constant" $\mathrm{H}_{0}$ proportional with the relative distance " $z$ " between object and observer and relative velocity " $v$ " between both objects and strong inhomogeneous gravitational fields (Black Holes). Until now, this is the only theory which explains the deviations in the "Hubble Constant", measured by the "Hubble Space Telescope" at distances larger than about $1000 \mathrm{Mpc}$.

\subsection{Data Availability}

All the Data and all the Calculations to provide evidence to "Quantum Light Theory" have been published in the 'Open Source Framework(OSF)': https://osf.io/gbn4p/

DOI: $10.31219 /$ osf.io/gbn4p

( https://doi.org/10.31219/osf.io/gbn4p )

(Calculations in Mathematica 11.0)', Page 1-33). 


\section{References}

[1] Geons; Wheeler; John Archibald; Phys. Rev. 97, 511, Issue 2, pages 511-526, Published 15 January 1955; Publisher: American Physical Scociety; DOI: 10.1103/PhysRev.97.511.

[2] Operational Resource Theory of Imaginarity; Kang-Da Wu, Tulja Varun Kondra, Swapan Rana, Carlo Maria Scandolo, Guo-Yong Xiang, Chuan-Feng Li, GuangCan Guo, and Alexander Streltsov; Phys. Rev. Lett. 126, 090401 - Published 1 March 2021.

[4] Z. K. Minev, S. O. Mundhada, S. Shankar, P. Reinhold, R. Gutiérrez-Jáuregui, R.J. Schoelkopf, N. Mirrahimi, H.J. Carmichael and M. H. Devoret; To catch and reverse a quantum jump mid-flight; Nature; 03 June 2019 equilibrium beyond Einstein 4-Dimensional, Kaluza-Klein 5-Dimensional and Superstring 10- and 11 Dimensional Curved Hyperspaces. https://www.nature.com/articles/s41586-019$1287-\mathrm{Z}$

[5] A. S. Stodolna, A. Rouzée, F. Lépine, S. Cohen, F. Robicheaux, A. Gijsbertsen, J. H. Jungmann, C. Bordas, and M. J. J. Vrakking. Hydrogen Atoms under Magnification: Direct Observation of the Nodal Structure of Stark States. Phys. Rev. Lett. 110, 213001 - Published 20 May 2013

[6] IBM Blog Research; A new effect in electromagnetism discovered - 150 years later; https://phys.org/news/2017-10effect-electromagnetism-years.html

[7] Abuter, R.; Amorim, A.; Anugu, N.; Bauböck, M.; Benisty, M.; Berger, J. P.; Blind, N.; Bonnet, H.; Brandner, W.; Buron, A.; Collin, C. (2018-07-01). "Detection of the gravitational redshift in the orbit of the star S2 near the Galactic centre massive black hole". Astronomy \& Astrophysics. DOI: https://doi.org/10.1051/0004$6361 / 201833718$ 
[8] "Gurzadyan, V.G., Stepanian, A. Hubble tension vs two flows. Eur. Phys. J. Plus 136, 235 (2021).

https://doi.org/10.1140/epip/s13360-021-01229-x

[9] "Hayley J. Macpherson et al", 2018, The Astrophysical Journal Letters, Volume 865, Number 1

[10] "M.L.Mc Clure, C.C.Dyerb, Anisotropy in the Hubble constant as observed in the HST extragalactic distance scale key project results,

https://doi.org/10.1016/j.newast.2007.03.005

[11] Antonio Enea Romano, Hubble trouble or Hubble bubble?, International Journal of Modern Physics D VOL. 27, NO. 09, https://doi.org/10.1142/S021827181850102X

[12] Kaya, Ali, Hubble's law and faster than light expansion speeds, American Journal of Physics 79, 1151 (2011); https://doi.org/10.1119/1.3625871

[13] Eleonora Di Valentino et all, In the Realm of the Hubble tension - a Review of Solutions, Classical and Quantum Gravity, DOI: 10.1088/1361-6382/ac086d [14] S.L. Parnovsky, Bias of the Hubble constant value caused by errors in galactic distance indicators, Ukr. J. Phys, https://arxiv.org/abs/2109.09645v2

[15] Stephen M. Feeney, Hiranya V. Peiris, Samaya M.

Nissanke, and Daniel J. Mortlock, Prospects for Measuring the Hubble Constant with Neutron-Star-Black-Hole Mergers Phys. Rev. Lett. 126, 171102 - Published 28 April 2021. [16] T. de Jaeger, B. E. Stahl, W. Zheng, A .V Filippenko, A. G. Riess, L. Galbany, A measurement of the Hubble constant from Type II supernovae, Monthly Notices of the Royal Astronomical Society, Volume 496, Issue 3, August 2020, https://doi.org/10.1093/mnras/staa1801

[17] Wendy L. Freedman, Measurements of the Hubble Constant: Tensions in Perspective, Department of Astronomy \& Astrophysics \& Kavli Institute for Cosmological Physics, University of Chicago, arxiv.org/pdf/2106.15656.pdf. 
[18] Licia Verde, Tommaso Treu and Adam G. Riess,

Tensions between the early and late Universe, Nat Astron 3, 891-895 (2019), https://doi.org/10.1038/s41550-019-0902-0 